\title{
Mechanism design for a solution to the tragedy of commons
}

\author{
Akira Yamada • Naoki Yoshihara
}

Received: 31 July 2003 / Accepted: 22 October 2007 / Published online: 4 January 2008

(C) The Author(s) 2007

\begin{abstract}
We consider the problem of the tragedy of commons in cooperative production economies, and propose a mechanism to resolve this tragedy, taking into account that the coordinator cannot perfectly monitor each agent's labor skill and each agent may have an incentive to overstate as well as understate his own skill. Even in such a situation, the mechanism implements the proportional solution (Roemer in Soc Philos Policy 6:74-92, 1989 and Roemer and Silvestre in J Econ Theory 59:426-444, 1993) in Nash and strong equilibria when it is played as a normal form game. Moreover, the mechanism triply implements the solution in Nash, subgame-perfect, and strong equilibria when it is played as a two-stage extensive form game.
\end{abstract}

Keywords Triple implementation · Proportional solution · Unknown and possibly overstated labor skills · Labor sovereignty

JEL Classification $\mathrm{C} 72 \cdot \mathrm{D} 51 \cdot \mathrm{D} 78 \cdot \mathrm{D} 82$

\begin{abstract}
We are greatly thankful to Semi Koray, William Thomson, and an anonymous referee of this journal for their concrete and helpful comments on improving the paper. An earlier version of this paper was presented at the annual meeting of the Japanese Economic Association held at Hitotsubashi University in October 2001 and at the Conference on Economic Design held at NYU in July 2002. We are grateful to Takehiko Yamato for his useful comments in the former conference. We are also thankful to Tatsuyoshi Saijo, Kotaro Suzumura, and Yoshikatsu Tatamitani for their kind comments.
\end{abstract}

\section{A. Yamada}

Faculty of Economics, Sapporo University, 7-3-1 Nishioka 3-jo, Toyohira-ku, Sapporo, Hokkaido 062-8520, Japan

e-mail: a-yamada@sapporo-u.ac.jp

N. Yoshihara $(\varangle)$

Institute of Economic Research, Hitotsubashi University, Naka 2-1, Kunitachi,

Tokyo 186-0004, Japan

e-mail: yosihara@ier.hit-u.ac.jp 


\section{Introduction}

The fact that resource allocation under free access to technology results in "overproduction" and inefficient Nash equilibria in cooperative production economies is best known as the "tragedy of commons". This paper provides a mechanism that can solve this tragedy. As a normative solution for the tragedy, we adopt the proportional solution (Roemer and Silvestre 1993) under joint ownership of the technology, which assigns Pareto efficient allocations, in which each agent's output consumption is proportional to his labor contribution. Then, we construct an incentive-compatible mechanism that implements the proportional solution.

There are some works on the implementation of the proportional solution, such as Suh (1995), Yoshihara (1999, 2000a), and Tian (2000), as well as of other social choice correspondences in production economies. ${ }^{1}$ However, in most of the literature on implementation in production economies, a nonnegligible problem of asymmetric information in the production process appears to be treated as a "black box." Under any mechanism, each agent is usually required to provide some information, and the outcome function assigns an allocation to each profile of agents' strategies. This implicitly assumes, in production economies with labor input, that the mechanism coordinator is authorized to make agents supply their labor hours consistent with the assigned allocation. ${ }^{2}$ This is because the original concern of implementation theory was in regard to adverse selection problems, and such a focus was valid whenever there was a decentralized resource allocation in exchange economies and/or production economies with no labor input. However, in production economies with labor input, this assumption is not realistic.

As an alternative, in this paper we assume that the coordinator is not authorized to make agents work as he pleases; the coordinator can monitor each agent's labor hours, but the coordinator cannot perfectly monitor each agent's effective labor contribution measured in efficiency units, since the coordinator is incapable of observing each agent's labor skill exercised in the production process. Thus, there may be an incentive for each agent to overstate or understate his own skill. ${ }^{3}$ Even under such a more realistic model of the tragedy of commons, the mechanism proposed in this paper can implement the solution.

This mechanism is a type of sharing mechanism: each agent can freely supply his labor hours, and the agent is asked to provide some information about his demand for

\footnotetext{
1 For example, Hurwicz et al. (1995), Hong (1995), and Tian (1999) for private ownership production economies with only private goods, Varian (1994) for production economies with the presence of an externality, and Kaplan and Wettstein (2000) and Corchón and Puy (2002) for cooperative production economies.

2 Roemer (1989) pointed out this assumption explicitly.

3 Tian (2000) constructed a mechanism that implements the proportional solution even if the coordinator does not know the agents' endowment vectors of commodities, under the assumption that agents cannot overstate their endowments. As Tian (2000) mentioned, such an assumption may be justified when endowments consist solely of material goods, since the coordinator can require agents to "place the claimed endowments on the table" (Hurwicz et al. 1995). In our setting where endowments are labor skills, such a requirement is no longer valid, since the coordinator may not inspect the amount of skills in advance of production.
} 
the consumption good and his skill. Then, the outcome function only distributes the produced output to agents, according to the given information and the record of their supply of labor hours. In this mechanism, there is no restriction on the strategy spaces that prohibits agents from understating or overstating their skills. We will demonstrate that this mechanism triply implements the proportional solution in Nash, strong Nash, and subgame-perfect equilibria.

The basic model of economies and sharing mechanisms is defined in Sect. 2. Section 3 provides a sharing mechanism that implements the proportional solution. Concluding remarks are in Sect. 4. All proofs are provided in the Appendix.

\section{The basic model}

There are two goods, one of which is an input (labor time) $x \in \mathbb{R}_{+}$to be used to produce the other good $y \in \mathbb{R}_{+} \cdot{ }^{4}$ There is a set $N=\{1, \ldots, n\}$ of agents, where $2 \leq n<+\infty$. Each agent $i^{\prime}$ s consumption is denoted by $z_{i}=\left(x_{i}, y_{i}\right)$, where $x_{i}$ denotes his labor time, and $y_{i}$ the amount of the output to be consumed by $i$. All agents face a common upper bound of labor time $\bar{x}$, where $0<\bar{x}<+\infty$, and so have the same consumption set $Z \equiv[0, \bar{x}] \times \mathbb{R}_{+}$. Each agent $i^{\prime}$ s preference is defined on $Z$ and represented by a utility function $u_{i}: Z \rightarrow \mathbb{R}$, which is continuous and quasiconcave on $Z$, and strictly monotonic (decreasing in labor time and increasing in the share of output) on $\stackrel{\circ}{Z} \equiv[0, \bar{x}) \times \mathbb{R}_{++}{ }^{5}$ We use $\mathcal{U}$ to denote the class of such utility functions. Each agent $i$ also has a labor skill which is represented by a positive real number $s_{i} \in \mathbb{R}_{++}$. The universal set of skills for all agents is denoted by $\mathcal{S}=\mathbb{R}_{++}$. The labor skill $s_{i} \in \mathcal{S}$ implies $i^{\prime}$ s effective labor supply per hour measured in efficiency units. Thus, if the agent's supply of labor time is $x_{i} \in[0, \bar{x}]$ and the agent's skill is $s_{i} \in \mathcal{S}$, then $s_{i} x_{i} \in \mathbb{R}_{+}$represents the agent's substantive contribution in labor supply to production. The production technology is a function $f: \mathbb{R}_{+} \rightarrow \mathbb{R}_{+}$, which is continuous, strictly increasing, concave with $f(0)=0$. We choose an arbitrary such production technology function $f$ and keep it fixed in the sequel. Thus, an economy is a pair of profiles $\boldsymbol{e} \equiv(\boldsymbol{u}, \boldsymbol{s})$ with $\boldsymbol{u}=\left(u_{i}\right)_{i \in N} \in \mathcal{U}^{n}$ and $\boldsymbol{s}=\left(s_{i}\right)_{i \in N} \in \mathcal{S}^{n}$. Denote the class of such economies by $\mathcal{E} \equiv \mathcal{U}^{n} \times \mathcal{S}^{n}$.

Given $s \in \mathcal{S}^{n}$, an allocation $z=\left(x_{i}, y_{i}\right)_{i \in N} \in Z^{n}$ is feasible for $\boldsymbol{s}$ if $\sum y_{i} \leq$ $f\left(\sum s_{i} x_{i}\right)$. We denote by $Z(s)$ the set of feasible allocations for $s \in \mathcal{S}^{n}$. An allocation $\boldsymbol{z}=\left(z_{i}\right)_{i \in N} \in Z^{n}$ is Pareto efficient for $\boldsymbol{e}=(\boldsymbol{u}, \boldsymbol{s}) \in \mathcal{E}$ if $\boldsymbol{z} \in Z(\boldsymbol{s})$ and there does not exist $z^{\prime}=\left(z_{i}^{\prime}\right)_{i \in N} \in Z(s)$ such that for all $i \in N, u_{i}\left(z_{i}^{\prime}\right) \geq u_{i}\left(z_{i}\right)$, and for some $i \in N, u_{i}\left(z_{i}^{\prime}\right)>u_{i}\left(z_{i}\right)$. The proportional solution (Roemer and Silvestre 1993) is a correspondence $P R: \mathcal{E} \rightarrow Z^{n}$ such that, for each $\boldsymbol{e}=(\boldsymbol{u}, \boldsymbol{s}) \in \mathcal{E}, P R(\boldsymbol{e})$ stands for the set of all allocations $z=\left(x_{i}, y_{i}\right)_{i \in N} \in Z^{n}$ which are Pareto efficient for $\boldsymbol{e}$ such that, for each $i \in N, y_{i}=\frac{s_{i} x_{i}}{\sum s_{j} x_{j}} f\left(\sum s_{j} x_{j}\right)$. An allocation $z \in Z^{n}$ is $P R$-optimal for $\boldsymbol{e} \in \mathcal{E}$ if $z \in P R(\boldsymbol{e})$.

\footnotetext{
4 The symbol $\mathbb{R}_{+}$denotes the set of non-negative real numbers.

5 The symbol $\mathbb{R}_{++}$denotes the set of positive real numbers.
} 


\subsection{Sharing mechanisms}

We are interested in mechanisms having the property of labor sovereignty (Kranich 1994; Yoshihara 2000b), ${ }^{6}$ which says that every agent can choose freely his own labor time. As such, we focus on the following types of mechanisms. For each $i \in N$, let his strategy space be $A_{i} \equiv M_{i} \times[0, \bar{x}]$, with generic element $\left(m_{i}, x_{i}\right)$. Note that here $M_{i}$ stands for an abstract general message space as in classical mechanisms, while the members of $[0, \bar{x}]$, which represent $i$ 's choice of labor time as part of his observable action, are also considered as a strategic variable for $i$. Let $A \equiv \times_{i \in N} A_{i}$. Let $w \in \mathbb{R}_{+}$be the total output the coordinator observes after production. Then, a sharing mechanism is a function $g: A \times \mathbb{R}_{+} \rightarrow \mathbb{R}_{+}^{n}$ such that for each $(\boldsymbol{m}, \boldsymbol{x}) \in A$ and each $w \in \mathbb{R}_{+}, g(\boldsymbol{m}, \boldsymbol{x}, w)=\boldsymbol{y}$ for some $\boldsymbol{y} \in \mathbb{R}_{+}^{n}$. A sharing mechanism $g$ is feasible if for each $(\boldsymbol{m}, \boldsymbol{x}) \in A$ and each $w \in \mathbb{R}_{+}, \sum g_{i}(\boldsymbol{m}, \boldsymbol{x}, w) \leq w$. We denote by $\mathcal{G}$ the class of all (feasible sharing) mechanisms. In the following discussion, we assume that the production technology function $f$ is known and the total output after production is observable to the coordinator. Thus, for each $s \in \mathcal{S}^{n}$ and each $\boldsymbol{x} \in[0, \bar{x}]^{n}, w=f\left(\sum s_{j} x_{j}\right)$ is known to the coordinator after production, without the true information about $s .{ }^{7}$ Then, $g \in \mathcal{G}$ implies that for each $\boldsymbol{s} \in \mathcal{S}^{n}$ and each $(\boldsymbol{m}, \boldsymbol{x}) \in A,\left(\boldsymbol{x}, g\left(\boldsymbol{m}, \boldsymbol{x}, f\left(\sum s_{j} x_{j}\right)\right)\right) \in$ $Z(s)$. In the following discussion, for each $g \in \mathcal{G}$, we simply write a value of $g$ as $g(\boldsymbol{m}, \boldsymbol{x})$ instead of $g\left(\boldsymbol{m}, \boldsymbol{x}, f\left(\sum s_{j} x_{j}\right)\right)$ except for when we define new mechanisms in $\mathcal{G}$.

Given $g \in \mathcal{G}$, a (feasible) sharing game is defined for each economy $\boldsymbol{e} \in \mathcal{E}$ as a non-cooperative game $(N, A, g, \boldsymbol{e})$. Fixing the set of players $N$ and their strategy sets $A$, we simply denote a feasible sharing game $(N, A, g, \boldsymbol{e})$ by $(g, \boldsymbol{e})$.

Given a profile $(\boldsymbol{m}, \boldsymbol{x}) \in A$, let $\left(m_{i}^{\prime}, \boldsymbol{m}_{-i}, x_{i}^{\prime}, \boldsymbol{x}_{-i}\right) \in A$ be another strategy profile that is obtained by replacing the $i$-th component $\left(m_{i}, x_{i}\right)$ of $(\boldsymbol{m}, \boldsymbol{x})$ with $\left(m_{i}^{\prime}, x_{i}^{\prime}\right)$. A profile $\left(\boldsymbol{m}^{*}, \boldsymbol{x}^{*}\right) \in A$ is a (pure-strategy) Nash equilibrium of $(g, \boldsymbol{e})$ if for each $i \in N$ and each $\left(m_{i}, x_{i}\right) \in A_{i}, u_{i}\left(x_{i}^{*}, g_{i}\left(\boldsymbol{m}^{*}, \boldsymbol{x}^{*}\right)\right) \geq u_{i}\left(x_{i}, g_{i}\left(m_{i}, \boldsymbol{m}_{-i}^{*}, x_{i}, \boldsymbol{x}_{-i}^{*}\right)\right)$. Let $N E(g, \boldsymbol{e})$ denote the set of Nash equilibria of $(g, \boldsymbol{e})$. An allocation $z=\left(x_{i}, y_{i}\right)_{i \in N} \in$ $Z^{n}$ is a Nash equilibrium allocation of $(g, e)$ if there exists $\boldsymbol{m} \in M$ such that $(\boldsymbol{m}, \boldsymbol{x}) \in N E(g, \boldsymbol{e})$ and $\boldsymbol{y}=g(\boldsymbol{m}, \boldsymbol{x})$, where $\boldsymbol{x}=\left(x_{i}\right)_{i \in N}$ and $\boldsymbol{y}=\left(y_{i}\right)_{i \in N}$. Let $N A(g, \boldsymbol{e})$ denote the set of Nash equilibrium allocations of $(g, \boldsymbol{e})$. A mechanism $g \in \mathcal{G}$ implements $P R$ in Nash equilibria if for each $\boldsymbol{e} \in \mathcal{E}, N A(g, \boldsymbol{e})=$ $P R(\boldsymbol{e})$.

A profile $\left(\boldsymbol{m}^{*}, \boldsymbol{x}^{*}\right) \in A$ is a (pure-strategy) strong (Nash) equilibrium of $(g, \boldsymbol{e})$ if for each $T \subseteq N$ and each $\left(m_{i}, x_{i}\right)_{i \in T} \in\left(A_{i}\right)_{i \in T}$, there exists $j \in T$ such that

\footnotetext{
6 The previous mechanisms such as Suh (1995), Yoshihara (1999,2000a), and Tian (2000) do not have this property.

7 Since the coordinator also knows $f$ and $\boldsymbol{x}$, he can figure out that the true skill profile belongs to the hyperplane $\left\{s \in \mathcal{S}^{n} \mid \boldsymbol{s} \cdot \boldsymbol{x}=f^{-1}(w)\right\}$. However, the exact location of the true skill profile in this hyperplane cannot be figured out. Note that, to see which of the feasible allocations are true $P R$-optimal allocations, one needs to know the information of the true skill profile.
} 


$$
u_{j}\left(x_{j}^{*}, g_{j}\left(\boldsymbol{m}^{*}, \boldsymbol{x}^{*}\right)\right) \geq u_{j}\left(x_{j}, g_{j}\left(\left(m_{i}, x_{i}\right)_{i \in T},\left(m_{k}^{*}, x_{k}^{*}\right)_{k \in T^{c}}\right)\right) .^{8}
$$

Let $S N E(g, \boldsymbol{e})$ denote the set of strong equilibria of $(g, \boldsymbol{e})$. An allocation $z=$ $\left(x_{i}, y_{i}\right)_{i \in N} \in Z^{n}$ is a strong equilibrium allocation of $(g, \boldsymbol{e})$ if there exists $\boldsymbol{m} \in M$ such that $(\boldsymbol{m}, \boldsymbol{x}) \in S N E(g, \boldsymbol{e})$ and $\boldsymbol{y}=g(\boldsymbol{m}, \boldsymbol{x})$. Let $S N A(g, \boldsymbol{e})$ denote the set of strong equilibrium allocations of $(g, e)$. A mechanism $g \in \mathcal{G}$ implements $P R$ in strong equilibria, if for each $\boldsymbol{e} \in \mathcal{E}, S N A(g, \boldsymbol{e})=P R(\boldsymbol{e})$. Moreover, a mechanism $g \in \mathcal{G}$ doubly implements $P R$ in Nash and strong equilibria if for each $\boldsymbol{e} \in \mathcal{E}$, $N A(g, \boldsymbol{e})=S N A(g, \boldsymbol{e})=P R(\boldsymbol{e})$.

\subsection{Timing problem in sharing mechanisms}

Before discussing our own mechanism, we should mention the timing problem of strategy-decision in real applications of mechanisms, which is particularly relevant to the case of production economies. Note that $\boldsymbol{m}$ and $\boldsymbol{x}$ represent different kinds of strategic actions: $\boldsymbol{m}$ is the list of agents' announcements of their private information, while $\boldsymbol{x}$ is their production activity from supplying labor time. Thus, there may be a difference between the point in time when $\boldsymbol{m}$ is announced and the time when $\boldsymbol{x}$ is exercised. It implies that there may be at least two polar cases of time sequence with regard to decision making: the agents may announce $\boldsymbol{m}$ before they engage in production, or they may announce $\boldsymbol{m}$ after supplying $\boldsymbol{x}$. The former enables each agent $i$ to choose his labor supply with knowledge of the announcements $\boldsymbol{m}$, whereas the latter enables each agent $i$ to choose $m_{i}$ with knowledge of the agents' actions $\boldsymbol{x}$ in the production process.

Thus, we should consider at least two types of two-stage game forms: Given $g \in \mathcal{G}$, the first two-stage extensive game form derived from $g$ is a feasible mechanism $\Gamma_{g}^{m \circ x}$ in which Stage 1 consists of selecting $\boldsymbol{m} \in M$, Stage 2 consists of selecting $\boldsymbol{x} \in[0, \bar{x}]^{n}$, and then $(\boldsymbol{x}, g(\boldsymbol{m}, \boldsymbol{x}))$ is the outcome. The second two-stage extensive game form derived from $g$ is a feasible mechanism $\Gamma_{g}^{x \circ m}$ in which Stage 1 consists of selecting $\boldsymbol{x} \in[0, \bar{x}]^{n}$, Stage 2 consists of selecting $\boldsymbol{m} \in M$, and then $(\boldsymbol{x}, g(\boldsymbol{m}, \boldsymbol{x}))$ is the outcome.

Given a two-stage game $\left(\Gamma_{g}^{m \circ x}, \boldsymbol{e}\right)$ and a strategy profile $\boldsymbol{m} \in M$ in Stage 1 , let $\left(\Gamma_{g}^{m \circ x}(\boldsymbol{m}), \boldsymbol{e}\right)$ be the corresponding Stage 2 subgame. A strategy mapping $\chi$ : $M \rightarrow[0, \bar{x}]^{n}$ is a function such that for each $\boldsymbol{m} \in M, \chi(\boldsymbol{m})$ is a strategy profile of the subgame $\left(\Gamma_{g}^{m \circ x}(\boldsymbol{m}), \boldsymbol{e}\right)$. Let $\mathcal{X}$ be the set of such mappings. A profile $\left(\boldsymbol{m}^{*}, \chi^{*}\right) \in$ $M \times \mathcal{X}$ is a (pure-strategy) subgame perfect (Nash) equilibrium of $\left(\Gamma_{g}^{m \circ x}, e\right)$ if for each $i \in N$, each $m_{i} \in M_{i}$, each $\chi \in \mathcal{X}$ with $\chi=\left(\chi_{i}, \chi_{-i}^{*}\right)$, and each $\boldsymbol{m} \in M$,

$$
\begin{aligned}
& \quad u_{i}\left(\chi_{i}^{*}\left(\boldsymbol{m}^{*}\right), g_{i}\left(\boldsymbol{m}^{*}, \chi^{*}\left(\boldsymbol{m}^{*}\right)\right)\right) \geq u_{i}\left(\chi_{i}^{*}\left(m_{i}, \boldsymbol{m}_{-i}^{*}\right), g_{i}\left(m_{i}, \boldsymbol{m}_{-i}^{*}, \chi^{*}\left(m_{i}, \boldsymbol{m}_{-i}^{*}\right)\right)\right) \\
& \text { and } u_{i}\left(\chi_{i}^{*}(\boldsymbol{m}), g_{i}\left(\boldsymbol{m}, \chi^{*}(\boldsymbol{m})\right)\right) \geq u_{i}\left(\chi_{i}(\boldsymbol{m}), g_{i}(\boldsymbol{m}, \boldsymbol{\chi}(\boldsymbol{m}))\right),
\end{aligned}
$$

\footnotetext{
${ }^{8}$ For each $T \subseteq N$, \#T denotes the number of agents in $T$. For each $T \subseteq N, T^{c}$ denotes the complement of $T$ in $N$.
} 
where $\chi_{i}^{*}(\boldsymbol{m})\left(\right.$ resp $\left.\cdot \chi_{i}(\boldsymbol{m})\right)$ is the $i$-th component of $\chi^{*}(\boldsymbol{m})($ resp $\cdot \boldsymbol{\chi}(\boldsymbol{m}))$ in Stage 2 subgame induced by the choice $\boldsymbol{m}$ in Stage 1.

Given a two-stage game $\left(\Gamma_{g}^{x \circ m}, e\right)$ and a strategy profile $x \in[0, \bar{x}]^{n}$ in Stage 1, let $\left(\Gamma_{g}^{x \circ m}(x), e\right)$ be the corresponding Stage 2 subgame. A strategy mapping $\boldsymbol{\mu}$ : $[0, \bar{x}]^{n} \rightarrow M$ is a function such that for each $\boldsymbol{x} \in[0, \bar{x}]^{n}, \boldsymbol{\mu}(\boldsymbol{x})$ is a strategy profile of the subgame $\left(\Gamma_{g}^{x \circ m}(\boldsymbol{x}), \boldsymbol{e}\right)$. Let $\mathcal{M}$ be the set of such mappings. A profile $\left(\boldsymbol{\mu}^{*}, \boldsymbol{x}^{*}\right) \in$ $\mathcal{M} \times[0, \bar{x}]^{n}$ is a (pure-strategy) subgame perfect (Nash) equilibrium of $\left(\Gamma_{g}^{x \circ m}, \boldsymbol{e}\right)$ if for each $i \in N$, each $x_{i} \in[0, \bar{x}]$, each $\mu \in \mathcal{M}$ with $\mu=\left(\mu_{i}, \mu_{-i}^{*}\right)$, and each $\boldsymbol{x} \in[0, \bar{x}]^{n}$,

$$
\begin{aligned}
u_{i}\left(x_{i}^{*}, g_{i}\left(\boldsymbol{\mu}^{*}\left(\boldsymbol{x}^{*}\right), \boldsymbol{x}^{*}\right)\right) & \geq u_{i}\left(x_{i}, g_{i}\left(\boldsymbol{\mu}^{*}\left(x_{i}, \boldsymbol{x}_{-i}^{*}\right), x_{i}, \boldsymbol{x}_{-i}^{*}\right)\right), \\
\text { and } u_{i}\left(x_{i}, g_{i}\left(\boldsymbol{\mu}^{*}(\boldsymbol{x}), \boldsymbol{x}\right)\right) & \geq u_{i}\left(x_{i}, g_{i}(\boldsymbol{\mu}(\boldsymbol{x}), \boldsymbol{x})\right)
\end{aligned}
$$

Let $S P E\left(\Gamma_{g}^{m \circ x}, \boldsymbol{e}\right)$ be the set of subgame perfect equilibria of $\left(\Gamma_{g}^{\boldsymbol{m} \circ \boldsymbol{x}}, \boldsymbol{e}\right)$. An allocation $z=\left(x_{i}, y_{i}\right)_{i \in N} \in Z^{n}$ is a subgame perfect equilibrium allocation of $\left(\Gamma_{g}^{m \circ x}, \boldsymbol{e}\right)$ if there exists $(\boldsymbol{m}, \chi) \in S P E\left(\Gamma_{g}^{\boldsymbol{m} \circ \boldsymbol{x}}, \boldsymbol{e}\right)$ such that $\chi(\boldsymbol{m})=\boldsymbol{x}$ and $\boldsymbol{y}=g(\boldsymbol{m}, \chi(\boldsymbol{m}))$. Let $S P A\left(\Gamma_{g}^{\boldsymbol{m} \circ \boldsymbol{x}}, \boldsymbol{e}\right)$ be the set of subgame perfect equilibrium allocations of $\left(\Gamma_{g}^{\boldsymbol{m} \circ \boldsymbol{x}}, \boldsymbol{e}\right)$. Given $g \in \mathcal{G}, \Gamma_{g}^{\boldsymbol{m} \circ \boldsymbol{x}}$ implements $P R$ in subgame perfect equilibria if for each $\boldsymbol{e} \in \mathcal{E}, S P A\left(\Gamma_{g}^{\boldsymbol{m} \circ \boldsymbol{x}}, \boldsymbol{e}\right)=P R(\boldsymbol{e})$. Given $g \in \mathcal{G}, \Gamma_{g}^{\boldsymbol{m} \circ \boldsymbol{x}}$ triply implements $P R$ in Nash, strong, and subgame perfect equilibria if for each $e \in \mathcal{E}$, $N A(g, \boldsymbol{e})=S N A(g, \boldsymbol{e})=S P A\left(\Gamma_{g}{ }^{\boldsymbol{m} \circ \boldsymbol{x}}, \boldsymbol{e}\right)=P R(\boldsymbol{e}) .{ }^{9}$ Parallel definitions apply to $\left(\Gamma_{g}^{x \circ m}, e\right)$.

\section{Implementation of the proportional solution}

In the following, we impose two additional assumptions.

Assumption 1 (boundary condition): $\forall i \in N, \forall z_{i} \in \stackrel{\circ}{Z}, \forall z_{i}^{\prime} \in[0, \bar{x}] \times\{0\}, u_{i}\left(z_{i}\right)>$ $u_{i}\left(z_{i}^{\prime}\right)$.

Assumption 2 The production function $f$ is continuously differentiable.

We denote by $f^{\prime}(x)$ the derivative of $f$ at $x$.

The message space $M$ of the mechanism in this paper is defined by $M \equiv \mathcal{S}^{n} \times \mathbb{R}_{+}^{n}$ with generic element $(\boldsymbol{\sigma}, \boldsymbol{y})$, where $\sigma=\left(\sigma_{i}\right)_{i \in N}$, in which $\sigma_{i}$ denotes $i$ 's reported skill, and $\boldsymbol{y}=\left(y_{i}\right)_{i \in N}$, in which $y_{i}$ denotes $i$ 's demand for output.

\footnotetext{
9 This definition contains some abuse of language, as the implementation in Nash and strong equilibria is achieved by the mechanism $(g, \boldsymbol{e})$, while it is $\Gamma_{g}^{m \circ x}$ that implements $P R$ in subgame perfect equilibria.
} 


\subsection{Nash and strong implementability}

In this subsection, we will set aside the timing problem of sharing mechanisms and propose a sharing mechanism as a normal form game form that implements $P R$ in Nash and strong equilibria. To propose our mechanism, let us introduce two feasible sharing mechanisms, defined as follows:

- $g^{\pi} \in \mathcal{G}$ is such that for each $(\boldsymbol{\sigma}, \boldsymbol{x}, \boldsymbol{y}) \in \mathcal{S}^{n} \times[0, \bar{x}]^{n} \times \mathbb{R}_{+}^{n}$, each $w \in \mathbb{R}_{+}$, and each $i \in N$,

$g_{i}^{\pi}(\boldsymbol{\sigma}, \boldsymbol{x}, \boldsymbol{y}, w)= \begin{cases}w & \text { if } \Pi\left(\boldsymbol{x}_{-i}\right) \neq \varnothing, x_{i}=\pi\left(\boldsymbol{x}_{-i}\right), \text { and } y_{i}>\max \left\{y_{j} \mid j \neq i\right\} \\ 0 & \text { otherwise }\end{cases}$ where $\Pi\left(\boldsymbol{x}_{-i}\right) \equiv\left\{\frac{x_{j}+\bar{x}}{2} \mid x_{j}<\bar{x}\right.$ for $\left.j \neq i\right\}$ and $\pi\left(\boldsymbol{x}_{-i}\right) \equiv \max \Pi\left(\boldsymbol{x}_{-i}\right)$.

- $g^{\sigma} \in \mathcal{G}$ is such that for each $(\boldsymbol{\sigma}, \boldsymbol{x}, \boldsymbol{y}) \in \mathcal{S}^{n} \times[0, \bar{x}]^{n} \times \mathbb{R}_{+}^{n}$, each $w \in \mathbb{R}_{+}$, and each $i \in N$, $g_{i}^{\sigma}(\boldsymbol{\sigma}, \boldsymbol{x}, \boldsymbol{y}, w)=\left\{\begin{array}{l}w \text { if } x_{i}=0, \text { and } \sigma_{i}>\sigma_{j} \text { for all } j \neq i \\ 0 \text { otherwise. }\end{array}\right.$

The mechanism $g^{\pi} \in \mathcal{G}$ assigns all of the produced output to only one agent who provides less than $\bar{x}$ of labor time, but the maximal positive amount among those who provide less than $\bar{x}$ of labor time, and reports a maximal amount of demand for the output, if there is such an agent at all. The mechanism $g^{\sigma} \in \mathcal{G}$ also assigns all of the produced output to only one agent who reports the highest labor skill and provides no labor time.

Given $(\boldsymbol{s}, \boldsymbol{x}, \boldsymbol{y})=\left(s_{i}, x_{i}, y_{i}\right)_{i \in N} \in \mathcal{S}^{n} \times Z^{n}$, let $P R(\boldsymbol{s}, \boldsymbol{x}, \boldsymbol{y})^{-1} \equiv\left\{\boldsymbol{u} \in \mathcal{U}^{n} \mid(\boldsymbol{x}, \boldsymbol{y}) \in\right.$ $P R(\boldsymbol{u}, \boldsymbol{s})\}$. If $P R(\boldsymbol{s}, \boldsymbol{x}, \boldsymbol{y})^{-1} \neq \varnothing$, then $(\boldsymbol{x}, \boldsymbol{y})$ should be a $P R$-optimal allocation for some economy with skill profile $s$. Let us call such an $(\boldsymbol{s}, \boldsymbol{x}, \boldsymbol{y})$ a $P R$ - consistent profile. Note that if for all $i \in N, y_{i}=\frac{s_{i} x_{i}}{\sum s_{k} x_{k}} f\left(\sum s_{k} x_{k}\right)$ and $(\boldsymbol{x}, \boldsymbol{y})$ is an interior allocation, then $P R(\boldsymbol{s}, \boldsymbol{x}, \boldsymbol{y})^{-1} \neq \varnothing$. Given $(\boldsymbol{s}, \boldsymbol{x}, \boldsymbol{y}) \in \mathcal{S}^{n} \times Z^{n}$, let $N(\boldsymbol{s}, \boldsymbol{x}, \boldsymbol{y}) \equiv$ $\left\{i \in N \mid \exists\left(s_{i}^{\prime}, x_{i}^{\prime}, y_{i}^{\prime}\right) \in \mathcal{S} \times Z\right.$ s.t. $\left.P R\left(s_{i}^{\prime}, x_{i}^{\prime}, y_{i}^{\prime}, \boldsymbol{s}_{-i}, \boldsymbol{x}_{-i}, \boldsymbol{y}_{-i}\right)^{-1} \neq \varnothing\right\}$. This $N(\boldsymbol{s}, \boldsymbol{x}, \boldsymbol{y})$ is the set of potential deviators under the profile $(\boldsymbol{s}, \boldsymbol{x}, \boldsymbol{y})$, since any $i \in$ $N(\boldsymbol{s}, \boldsymbol{x}, \boldsymbol{y})$ can constitute a $P R$-consistent profile with the others' fixed strategies by switching his strategy from $\left(s_{i}, x_{i}, y_{i}\right)$. Given $(\boldsymbol{s}, \boldsymbol{x}, \boldsymbol{y}) \in \mathcal{S}^{n} \times Z^{n}$ and $i \in N(\boldsymbol{s}, \boldsymbol{x}, \boldsymbol{y})$, let $\mathcal{S}^{i}(\boldsymbol{s}, \boldsymbol{x}, \boldsymbol{y}) \equiv\left\{s_{i}^{\prime} \in \mathcal{S} \mid \exists\left(x_{i}^{\prime}, y_{i}^{\prime}\right) \in Z\right.$ s.t. $\left.P R\left(s_{i}^{\prime}, x_{i}^{\prime}, y_{i}^{\prime}, \boldsymbol{s}_{-i}, \boldsymbol{x}_{-i}, \boldsymbol{y}_{-i}\right)^{-1} \neq \varnothing\right\}$. Note that $\mathcal{S}^{i}(\boldsymbol{s}, \boldsymbol{x}, \boldsymbol{y})$ is closed and bounded from below, or otherwise, $\mathcal{S}^{i}(\boldsymbol{s}, \boldsymbol{x}, \boldsymbol{y})=\mathcal{S}$. The latter case occurs if and only if there exists $b \in \mathbb{R}_{++}$such that $\sum_{k \neq i} s_{k} x_{k}<b$ and $f$ is linear on $[0, b]$.

Given $(\boldsymbol{s}, \boldsymbol{x}, \boldsymbol{y}) \in \mathcal{S}^{n} \times Z^{n}$ and $i \in N(\boldsymbol{s}, \boldsymbol{x}, \boldsymbol{y})$, let $\left(\widehat{s}_{i}, \widehat{x}_{i}, \widehat{y}_{i}\right) \in \mathcal{S} \times Z$ be defined by $\widehat{s}_{i}=\arg \min _{s_{i}^{\prime} \in \mathcal{S}^{i}(s, x, y)}\left|s_{i}^{\prime}-s_{i}\right|, 0<\widehat{x}_{i}<\bar{x}$, and $\widehat{y}_{i}$ with $\widehat{y}_{i}+\sum_{j \neq i} y_{j}=$ $f\left(\sum_{j \neq i} s_{j} x_{j}+\widehat{s}_{i} \widehat{x}_{i}\right)$ and $\frac{\sum_{j \neq i} y_{j}}{\widehat{y}_{i}}=\frac{\sum_{j \neq i} s_{j} x_{j}}{\widehat{s}_{i} \widehat{x}_{i}}$. Then, $P R\left(\widehat{s}_{i}, \widehat{x}_{i}, \widehat{y}_{i}, \boldsymbol{s}_{-i}, \boldsymbol{x}_{-i}\right.$, $\left.\boldsymbol{y}_{-i}\right)^{-1} \neq \varnothing$. Note that such $\left(\widehat{s}_{i}, \widehat{x}_{i}, \widehat{y}_{i}\right)$ is well-defined: first, $\widehat{s}_{i}$ is uniquely determined, since if $s_{i} \in \mathcal{S}^{i}(\boldsymbol{s}, \boldsymbol{x}, \boldsymbol{y})$, then $\widehat{s}_{i}=s_{i}$, whereas if $s_{i} \notin \mathcal{S}^{i}(\boldsymbol{s}, \boldsymbol{x}, \boldsymbol{y})$, then $\mathcal{S}^{i}(\boldsymbol{s}, \boldsymbol{x}, \boldsymbol{y})$ is bounded from below and $\widehat{s}_{i}=\min \mathcal{S}^{i}(\boldsymbol{s}, \boldsymbol{x}, \boldsymbol{y})$. Second, once $\widehat{s}_{i}$ is uniquely determined, then the other agents' strategies together with $\widehat{s}_{i}$ give us the information about $i$ 's potential consumption vector $\left(\widehat{x}_{i}, \widehat{y}_{i}\right)$ by the proportionality of the $P R$-optimal allocation. 
We introduce $g^{*} \in \mathcal{G}$ which works in each given $w \in \mathbb{R}_{+}$as follows: Let any $(\boldsymbol{\sigma}, \boldsymbol{x}, \boldsymbol{y})=\left(\sigma_{i}, x_{i}, y_{i}\right)_{i \in N} \in \mathcal{S}^{n} \times[0, \bar{x}]^{n} \times \mathbb{R}_{+}^{n}$ be given.

Rule 1 If $f\left(\sum \sigma_{k} x_{k}\right)=w$, then

1-1: if $P R(\sigma, x, y)^{-1} \neq \varnothing$, then $g^{*}(\sigma, x, y, w)=\boldsymbol{y}$,

1-2: if $P R(\sigma, \boldsymbol{x}, \boldsymbol{y})^{-1}=\varnothing$, and $N(\boldsymbol{\sigma}, \boldsymbol{x}, \boldsymbol{y}) \neq \varnothing$, then

1-2-1: if \#N $(\boldsymbol{\sigma}, \boldsymbol{x}, \boldsymbol{y})>1$, then $g^{*}(\boldsymbol{\sigma}, \boldsymbol{x}, \boldsymbol{y}, w)=\mathbf{0}$,

1-2-2: if $N(\boldsymbol{\sigma}, \boldsymbol{x}, \boldsymbol{y})=\{j\}$ for some $j \in N$, then

$g_{j}^{*}(\boldsymbol{\sigma}, \boldsymbol{x}, \boldsymbol{y}, w)=\min \left\{\max \left\{0, \widehat{y}_{j}+\widehat{s}_{j} \cdot f^{\prime}\left(\sum_{k \neq j} \sigma_{k} x_{k}+\widehat{s}_{j} \widehat{x}_{j}\right) \cdot\left(x_{j}-\hat{x}_{j}\right)\right\}, w\right\}$ and $g_{i}^{*}(\boldsymbol{\sigma}, \boldsymbol{x}, \boldsymbol{y}, w)=0$ for any $i \neq j$, where $P R\left(\widehat{s}_{j}, \widehat{x}_{j}, \widehat{y}_{j}, \boldsymbol{\sigma}_{-j}, \boldsymbol{x}_{-j}, \boldsymbol{y}_{-j}\right)^{-1} \neq \varnothing$, 1-3: in any other case, $g^{*}(\boldsymbol{\sigma}, \boldsymbol{x}, \boldsymbol{y}, w)=g^{\pi}(\boldsymbol{\sigma}, \boldsymbol{x}, \boldsymbol{y}, w)$.

Rule 2 If $f\left(\sum \sigma_{k} x_{k}\right) \neq w$, then $g^{*}(\boldsymbol{\sigma}, \boldsymbol{x}, \boldsymbol{y}, w)=g^{\sigma}(\boldsymbol{\sigma}, \boldsymbol{x}, \boldsymbol{y}, w)$.

First, $g^{*}$ computes the expected output $f\left(\sum \sigma_{k} x_{k}\right)$ from the data $(\boldsymbol{\sigma}, \boldsymbol{x}, \boldsymbol{y})$ and compares this with the real output $w$. In the case where these two values coincide, if $(\boldsymbol{\sigma}, \boldsymbol{x}, \boldsymbol{y})$ is $P R$-consistent, then $g^{*}$ gives the agents their desired $\boldsymbol{y}$ under Rule 1-1; if $(\boldsymbol{\sigma}, \boldsymbol{x}, \boldsymbol{y})$ is not $P R$-consistent, and there exists at least one potential deviator, say $j$, then $g^{*}$ gives him at most a share of outcome available in the budget set with the supporting price $\widehat{s}_{j} \cdot f^{\prime}\left(\sum_{k \neq j} \sigma_{k} x_{k}+\widehat{s}_{j} \widehat{x}_{j}\right)$, while $g^{*}$ gives nothing to any other agents under Rule 1-2; for any other case, $g^{*}$ applies $g^{\pi}$ under Rule 1-3. Finally, if $f\left(\sum \sigma_{k} x_{k}\right)$ and $w$ are different, then $g^{*}$ applies $g^{\sigma}$ under Rule 2.

It is easy to see that $g^{*}$ is forthright (Saijo et al. 1996) and satisfies best response property (Jackson et al. 1994). Moreover, $g^{*}$ is a quantity type, and so self-relevant (Hurwicz 1960). It is also easy to check that $g^{*}$ is feasible.

Let us briefly explain how $g^{*}$ induces true information about skills. This $g^{*}$ has to distribute the total output $f\left(\sum s_{k} x_{k}\right)$ according to $(\boldsymbol{\sigma}, \boldsymbol{x}, \boldsymbol{y})$, where $\left(s_{k}\right)_{k \in N}$ stands for the true skill profile and the coordinator cannot know whether $\sigma=s$ or not. If $f\left(\sum \sigma_{k} x_{k}\right) \neq f\left(\sum s_{k} x_{k}\right)$, however, then clearly $\sigma \neq s$, and there is at least one agent, say $j \in N$, such that $\sigma_{j} \neq s_{j}$ and $x_{j}>0$. Then, this agent is definitely punished under Rule 2. Next, consider the case where $f\left(\sum \sigma_{k} x_{k}\right)=f\left(\sum s_{k} x_{k}\right)$ but $\sigma \neq s$. Then, there are either at least two agents $i, j \in N$ such that $\sigma_{i} \neq s_{i}$, $\sigma_{j} \neq s_{j}, x_{i}>0$, and $x_{j}>0$, or else there exists an agent $j \in N$ such that $\sigma_{j} \neq s_{j}$ and $x_{j}=0$. If the latter case is applied, then the agents such as $j$ will be punished under Rule 1-2 or Rule 1-3. If the former case is applied, then one of the agents, $j \in N$, with $\sigma_{j} \neq s_{j}$ can induce Rule 2 by switching from $x_{j}>0$ to $x_{j}^{\prime}=0$, together with announcing a higher number $\sigma_{j}^{\prime} \neq s_{j}$ than any other $\sigma_{-j}$. Thus, this case may not correspond to an equilibrium. The following lemma actually confirms this insight:

Lemma 1 Let $g^{*} \in \mathcal{G}$ be given. Given $(\boldsymbol{u}, \boldsymbol{s}) \in \mathcal{E}$, let $(\boldsymbol{\sigma}, \boldsymbol{x}, \boldsymbol{y}) \in \mathcal{S}^{n} \times[0, \bar{x}]^{n} \times \mathbb{R}_{+}^{n}$ be a Nash equilibrium of $\left(g^{*}, \boldsymbol{u}, \boldsymbol{s}\right)$ such that $f\left(\sum \sigma_{k} x_{k}\right)=f\left(\sum s_{k} x_{k}\right)$. Then, it follows that for all $i \in N$ with $x_{i}>0, \sigma_{i}=s_{i}$.

Now, we examine the performance of $g^{*}$. 
Theorem 1 Let Assumptions 1 and 2 hold. Now the mechanism $g^{*}$ implements $P R$ in Nash and strong equilibria.

Note that $g^{*}$ works even in economies of two agents.

\subsection{Implementation of the proportional solution with the timing problem}

Because of the timing problem discussed in Sect. 2.2, $g^{*}$ may be played as $\Gamma_{g^{*}}^{(\sigma, y) \circ x}$ or $\Gamma_{g^{*}}^{x}(\sigma, y)$. In this situation, the coordinator may not know in advance the information structure of the two-stage game induced by $\Gamma_{g^{*}}^{(\sigma, y) \circ x}$ or $\Gamma_{g^{*}}^{x \circ(\sigma, y)}$, even if the coordinator has control over the number of stages in the mechanism: this information structure among agents may be characterized as perfect information, or as complete but imperfect information about Stage 1. If the game is played as one with perfect information (resp. complete but imperfect information), we should consider subgameperfect equilibria (resp. Nash equilibria) in the two-stage game. For instance, let us assume that the stage of announcing $(\boldsymbol{\sigma}, \boldsymbol{y})$ to the coordinator is in advance of production, in which $\boldsymbol{x}$ is supplied, and the information $(\boldsymbol{\sigma}, \boldsymbol{y})$ is not made public by the coordinator until the end of the production process. If this is effectively enforced, then the coordinator may be concerned with Nash and strong implementation only. Such a scenario implicitly assumes that the coordinator can effectively obstruct any private communication regarding $(\boldsymbol{\sigma}, \boldsymbol{y})$ among agents until the end of the production process. However, the coordinator may not be able to effectively wield this power, and agents could privately communicate with each other regarding $(\boldsymbol{\sigma}, \boldsymbol{y})$. This is one case that the coordinator cannot control the information structure among agents. In such a situation, the double implementability by $\Gamma_{g^{*}}^{(\sigma, y) \circ x}\left(\operatorname{resp} . \Gamma_{g^{*}}^{x \circ(\sigma, y)}\right)$ in Nash and subgame-perfect equilibria would be strongly attractive, since it keeps the desirable performance of the mechanism without relying on the information structure among agents:

Theorem 2 Let Assumptions 1 and 2 hold. Now the mechanism $\Gamma_{g^{*}}^{(\boldsymbol{\sigma}, \boldsymbol{y}) \circ \boldsymbol{x}}$ doubly implements $P R$ in Nash and subgame-perfect equilibria.

Theorem 3 Let Assumptions 1 and 2 hold. Now the mechanism $\Gamma_{g^{*}}^{x \circ(\boldsymbol{\sigma}, \boldsymbol{y})}$ doubly implements $P R$ in Nash and subgame-perfect equilibria.

By the three theorems discussed above, we can summarize as follows:

Corollary Let Assumptions 1 and 2 hold. Now both of the mechanisms $\Gamma_{g^{*}}^{(\sigma, y) \circ x}$ and $\Gamma_{g^{*}}^{x \circ(\sigma, y)}$ respectively triply implement $P R$ in Nash, subgame-perfect, and strong equilibria.

This result implies that $g^{*}$ implements $P R$ even if it permits each agent various kinds of freedom: the agent may choose freely his own supply of labor time; the agent is permitted to overstate his labor skill; the agent can behave unilaterally or coalitionally; 
Table 1 Performance of mechanisms implementing solutions for the tragedy of commons

\begin{tabular}{|c|c|c|c|c|c|c|c|}
\hline & $\begin{array}{l}\text { Suh } \\
\text { (1995) }\end{array}$ & $\begin{array}{l}\text { Yoshihara } \\
(1999)\end{array}$ & $\begin{array}{l}\text { Yoshihara } \\
(2000)\end{array}$ & $\begin{array}{l}\text { Kaplan- } \\
\text { Wettstein } \\
(2000)\end{array}$ & $\begin{array}{l}\text { Tian } \\
(2000)\end{array}$ & $\begin{array}{l}\text { Corchon- } \\
\text { Puy } \\
(2002)\end{array}$ & $\begin{array}{l}\text { Our } \\
\text { mechanism }\end{array}$ \\
\hline \multirow{3}{*}{$\begin{array}{c}\text { Equilibrium } \\
\text { notions* }\end{array}$} & $N A$ & $N A$ & $N A$ & $S P A$ & $N A$ & $N A$ & $N A$ \\
\hline & SNA & SNA & SNA & & SNA & & SNA \\
\hline & $U N A$ & $U N A$ & & & & & $S P A$ \\
\hline \# of goods & 2 & 2 & 2 or more & 2 & 2 or more & 2 & 2 \\
\hline \# of agents & 2 or more & 3 or more & 3 or more & 3 or more & 2 or more & 3 or more & 2 or more \\
\hline $\begin{array}{l}\text { Permission of } \\
\text { overstatement } \\
\text { of skills }\end{array}$ & No & No & No & No & No & No & Yes \\
\hline $\begin{array}{l}\text { Permission of } \\
\text { understatement } \\
\text { of skills }\end{array}$ & No & No & No & No & Yes & No & Yes \\
\hline Labor sovereignty & No & No & No & No & No & No & Yes \\
\hline Feasibility & Yes & Yes & Yes & Yes & Yes & Yes & Yes \\
\hline Self-relevancy & No & Yes & Yes & No & No & No & Yes \\
\hline $\begin{array}{l}\text { Best response } \\
\text { property }\end{array}$ & No & Yes & Yes & No & No & Yes & Yes \\
\hline Forthrightness & No & Yes & Yes & Yes & Yes & Yes & Yes \\
\hline Balancedness & No & Yes & Yes & No & No & Yes & No \\
\hline Continuity & No & No & No & No & Yes & No & No \\
\hline
\end{tabular}

* NA, SNA, UNA, and SPA mean Nash implementability, strong Nash implementability, undominated Nash implementability, and subgame-perfect implementability respectively

and the agent can behave strong-rationally, as in the subgame-perfect response, or weak-rationally, as in the Nash-like response. ${ }^{10}$

\section{Concluding remarks}

We have proposed a feasible sharing mechanism that triply implements the proportional solution in Nash, subgame-perfect, and strong equilibria, even when agents can understate or overstate their labor skills. The performance of our mechanism is summarized in Table 1, which provides a comparison with other relevant mechanisms.

\footnotetext{
${ }^{10}$ Let us point out this more precisely. Note that intermediate situations may happen where some agents behave unilaterally, and others coalitionally. For instance, let $\mathcal{H}$ be a subset of the power set of $N$, which is the set of admissible coalitions, and consider an equilibrium relative to $\mathcal{H}$, i.e., message-action profiles upon which no coalition in $\mathcal{H}$ can improve. When $\mathcal{H}$ stands for the set of all singletons, the equilibrium relative to $\mathcal{H}$ becomes the Nash equilibrium, while it becomes the strong equilibrium in case $\mathcal{H}$ is the set of all coalitions. The problem is that the mechanism coordinator may not expect what equilibrium notions the agents choose in the play of the game, since he has no information about the structure of $\mathcal{H}$. However, once a doubly-implementing mechanism is provided, such a difficulty is resolved, which gives us a motivation for the double implementation in Nash and strong equilibria.
} 
As revealed in Table 1, our mechanism has two undesirable features. First, it lacks continuity. Second, the mechanism fails to meet balancedness or nonwastefulness. One reason of the second undesirability is that the mechanism permits agents to both overstate and understate their labor skills. Thus, it is difficult to find the deviator when only aggregate information $\left(f\left(\sum \sigma_{k} x_{k}\right)\right.$ and $\left.f\left(\sum s_{k} x_{k}\right)\right)$ is available. Therefore, the mechanism basically punishes all agents when there is a deviator. The other reason is that this mechanism is characterized by labor sovereignty. The labor sovereignty mechanism should accept a profile of the agents' choice of labor time as an outcome, even when it may constitute a nondesirable allocation. Thus, if the mechanism needs to punish potential deviators, this is only possible by reducing their share of output, which leads to the violation of balancedness. We surmise that there may be a trade-off between labor sovereignty and balancedness. However, it remains an open question whether or not there exists a mechanism that satisfies both labor sovereignty and balancedness, and at the same time implements the proportional solution.

\section{Appendix}

Proof of Lemma 1 Suppose there exists $j \in N$ with $\sigma_{j} \neq s_{j}$ and $x_{j}>0$. Let $N_{L}(\sigma)$ be the set of such $j$. Since $f\left(\sum \sigma_{k} x_{k}\right)=f\left(\sum s_{k} x_{k}\right), N_{L}(\sigma)$ is not a singleton. Moreover, for each $j \in N_{L}(\sigma), y_{j}^{\prime}=f\left(\sum_{i \neq j} s_{i} x_{i}\right)>0$ by $\sigma_{j}^{\prime}>\max \left\{\sigma_{i} \mid i \neq j\right\}$ and $x_{j}^{\prime}=0$ under Rule 2 . Note that

$$
\begin{aligned}
& \sum_{j \in N_{L}(\boldsymbol{\sigma})} y_{j}^{\prime}=\sum_{j \in N_{L}(\boldsymbol{\sigma})} f\left(\sum_{i \neq j} s_{i} x_{i}\right) \\
& =\sum_{j \in N_{L}(\boldsymbol{\sigma})} f\left(\sum_{i \in N_{L}(\boldsymbol{\sigma}) \backslash\{j\}} s_{i} x_{i}+\sum_{i \notin N_{L}(\boldsymbol{\sigma})} s_{i} x_{i}\right) \\
& \geq \sum_{j \in N_{L}(\sigma)} f\left(s_{j} x_{j}+\sum_{i \notin N_{L}(\sigma)} s_{i} x_{i}\right) \\
& \text { (since } N_{L}(\sigma) \text { is not a singleton) } \\
& \geq f\left(\sum_{j \in N_{L}(\boldsymbol{\sigma})}\left(s_{j} x_{j}+\sum_{i \notin N_{L}(\boldsymbol{\sigma})} s_{i} x_{i}\right)\right) \\
& \text { (since } f \text { is concave and } f(0) \geq 0 \text { ) } \\
& \geq f\left(\sum_{j \in N_{L}(\boldsymbol{\sigma})} s_{j} x_{j}+\sum_{i \notin N_{L}(\boldsymbol{\sigma})} s_{i} x_{i}\right) \\
& \geq \sum_{j \in N_{L}(\boldsymbol{\sigma})} y_{j} \equiv \sum_{j \in N_{L}(\boldsymbol{\sigma})} g_{j}^{*}(\boldsymbol{\sigma}, \boldsymbol{x}, \boldsymbol{y}) \text {. }
\end{aligned}
$$


If there exists $j \in N_{L}(\boldsymbol{s})$ such that $y_{j}^{\prime}>y_{j}$, then $j$ has an incentive to switch from $x_{j}$ to $x_{j}^{\prime}=0$ and report $\sigma_{j}^{\prime}>\max \left\{\sigma_{i} \mid i \neq j\right\}$. If $y_{j}^{\prime}=y_{j}$ for each $j \in N_{L}(\sigma)$, then each $j \in N_{L}(\sigma)$ has an incentive to switch from $x_{j}$ to $x_{j}^{\prime}=0$, since $u_{j}\left(x_{j}^{\prime}, y_{j}^{\prime}\right)=$ $u_{j}\left(0, y_{j}\right)>u_{j}\left(x_{j}, y_{j}\right)$ by the strict monotonicity of utility functions. Thus, in any case, it contradicts the fact that $(\boldsymbol{\sigma}, \boldsymbol{x}, \boldsymbol{y})$ is a Nash equilibrium.

Proof of Theorem 1 (1) Show $P R(\boldsymbol{u}, \boldsymbol{s}) \subseteq N A\left(g^{*}, \boldsymbol{u}, \boldsymbol{s}\right)$ for each $(\boldsymbol{u}, \boldsymbol{s}) \in \mathcal{E}$. Let $\boldsymbol{z}=(\boldsymbol{x}, \boldsymbol{y}) \in P R(\boldsymbol{u}, \boldsymbol{s})$. If the strategy profile is $(\boldsymbol{s}, \boldsymbol{x}, \boldsymbol{y}) \in \mathcal{S}^{n} \times[0, \bar{x}]^{n} \times \mathbb{R}_{+}^{n}$, then $g^{*}(\boldsymbol{s}, \boldsymbol{x}, \boldsymbol{y})=\boldsymbol{y}$ by Rule 1-1. Since $\boldsymbol{s} \gg \boldsymbol{0}$ and $\boldsymbol{z}$ is an efficient proportional allocation, Assumption 1 implies $\boldsymbol{x} \gg \boldsymbol{0}$ and $g_{i}^{*}(\boldsymbol{s}, \boldsymbol{x}, \boldsymbol{y})>0$ for each $i \in N$. Suppose that $j \in N$ deviates from $\left(s_{j}, x_{j}, y_{j}\right)$ to $\left(\sigma_{j}^{\prime}, x_{j}^{\prime}, y_{j}^{\prime}\right) \in \mathcal{S} \times[0, \bar{x}] \times \mathbb{R}_{+}$.

Note first that the deviation cannot induce Rule 1-3. If the deviation results in Rule 1-2-1, then $g_{j}^{*}\left(\sigma_{j}^{\prime}, x_{j}^{\prime}, y_{j}^{\prime}, \boldsymbol{s}_{-j}, \boldsymbol{x}_{-j}, \boldsymbol{y}_{-j}\right)=0$. If the deviation induces Rule 2 , then $x_{j}^{\prime}>0$. Hence, $g_{j}^{*}\left(\sigma_{j}^{\prime}, x_{j}^{\prime}, y_{j}^{\prime}, \boldsymbol{s}_{-j}, \boldsymbol{x}_{-j}, \boldsymbol{y}_{-j}\right)=0$ under Rule 2.

If the deviation induces Rule 1-2-2 with $\sigma_{j}^{\prime} \neq s_{j}$, then $x_{j}^{\prime}=0$. Thus, $g_{j}^{*}\left(\sigma_{j}^{\prime}, x_{j}^{\prime}, y_{j}^{\prime}, \boldsymbol{s}_{-j}, \boldsymbol{x}_{-j}, \boldsymbol{y}_{-j}\right) \leq \widehat{y}_{j}-\widehat{s}_{j} \widehat{x}_{j} \cdot f^{\prime}\left(\sum_{i \neq j} s_{i} x_{i}+\widehat{s}_{j} \widehat{x}_{j}\right)$ by some $\left(\widehat{s}_{j}, \widehat{x}_{j}, \widehat{y}_{j}\right) \in \mathcal{S} \times[0, \bar{x}] \times \mathbb{R}_{+}$such that $P R\left(\widehat{s}_{j}, \widehat{x}_{j}, \widehat{y}_{j}, \boldsymbol{s}_{-j}, \boldsymbol{x}_{-j}, \boldsymbol{y}_{-j}\right)^{-1} \neq \varnothing$. Suppose $f\left(\sum_{i \neq j} s_{i} x_{i}+\widehat{s}_{j} \widehat{x}_{j}\right) \neq f\left(\sum_{i \neq j} s_{i} x_{i}+s_{j} x_{j}\right)$. Since $P R(\boldsymbol{s}, \boldsymbol{x}, \boldsymbol{y})^{-1} \neq \varnothing$, $\frac{f\left(\sum_{i \neq j} s_{i} x_{i}+\widehat{s}_{j} \widehat{x}_{j}\right)}{\sum_{i \neq j} s_{i} x_{i}+\widehat{s}_{j} \widehat{x}_{j}}=\frac{\sum_{i \neq j} y_{i}}{\sum_{i \neq j} s_{i} x_{i}}=\frac{f\left(\sum_{i \neq j} s_{i} x_{i}+s_{j} x_{j}\right)}{\sum_{i \neq j} s_{i} x_{i}+s_{j} x_{j}}$. Since $f$ is concave, $f$ must be linear on a closed interval $\left[0, \max \left\{\sum_{i \neq j} s_{i} x_{i}+\widehat{s}_{j} \widehat{x}_{j}, \sum_{i \neq j} s_{i} x_{i}+s_{j} x_{j}\right\}\right]$. Hence, $\widehat{y}_{j}-\widehat{s}_{j} \widehat{x}_{j} \cdot f^{\prime}\left(\sum_{i \neq j} s_{i} x_{i}+\widehat{s}_{j} \widehat{x}_{j}\right)=0$. Thus, $g_{j}^{*}\left(\sigma_{j}^{\prime}, x_{j}^{\prime}, y_{j}^{\prime}, \boldsymbol{s}_{-j}, \boldsymbol{x}_{-j}, \boldsymbol{y}_{-j}\right) \leq 0$. Next, suppose that $f\left(\sum_{i \neq j} s_{i} x_{i}+\widehat{s}_{j} \widehat{x}_{j}\right)=f\left(\sum_{i \neq j} s_{i} x_{i}+s_{j} x_{j}\right)$. This implies $\widehat{s}_{j} \widehat{x}_{j}=s_{j} x_{j}$ and $\widehat{y}_{j}=y_{j}$. Thus, $g_{j}^{*}\left(\sigma_{j}^{\prime}, x_{j}^{\prime}, y_{j}^{\prime}, \boldsymbol{s}_{-j}, \boldsymbol{x}_{-j}, \boldsymbol{y}_{-j}\right) \leq \widehat{y}_{j}-\widehat{s}_{j} \widehat{x}_{j}$. $f^{\prime}\left(\sum_{i \neq j} s_{i} x_{i}+\widehat{s}_{j} \widehat{x}_{j}\right)=y_{j}-s_{j} x_{j} \cdot f^{\prime}\left(\sum s_{k} x_{k}\right)$.

Consider the case where the deviation induces Rule 1-2-2 with $\sigma_{j}^{\prime}=s_{j}$. Since $\frac{f\left(\sum_{i \neq j} s_{i} x_{i}+s_{j} \widehat{x}_{j}\right)}{\sum_{i \neq j} s_{i} x_{i}+s_{j} \widehat{x}_{j}}=\frac{\sum_{i \neq j} y_{i}}{\sum_{i \neq j} s_{i} x_{i}}=\frac{f\left(\sum_{i \neq j} s_{i} x_{i}+s_{j} x_{j}\right)}{\sum_{i \neq j} s_{i} x_{i}+s_{j} x_{j}}$ by $\operatorname{PR}(\boldsymbol{s}, \boldsymbol{x}, \boldsymbol{y})^{-1} \neq \varnothing$, $f^{\prime}\left(\sum_{i \neq j} s_{i} x_{i}+s_{j} \widehat{x}_{j}\right)=f^{\prime}\left(\sum_{i \neq j} s_{i} x_{i}+s_{j} x_{j}\right)$. Thus, $g_{j}^{*}\left(s, x_{j}^{\prime}, \boldsymbol{x}_{-j}, y_{j}^{\prime}, \boldsymbol{y}_{-j}\right) \leq$ $y_{j}+s_{j} \cdot f^{\prime}\left(\sum s_{k} x_{k}\right) \cdot\left(x_{j}^{\prime}-x_{j}\right)$.

Next, if the deviation induces Rule 1-1, then $\sigma_{j}^{\prime}=s_{j}$. This is because $\sigma_{j}^{\prime} \neq s_{j}$ implies $x_{j}^{\prime}=0$ under Rule 1-1, which contradicts $\operatorname{PR}\left(\sigma_{j}^{\prime}, x_{j}^{\prime}, y_{j}^{\prime}, \boldsymbol{s}_{-j}, \boldsymbol{x}_{-j}, \boldsymbol{y}_{-j}\right)^{-1} \neq$ $\varnothing$ by Assumption 1. However, if the deviation induces Rule 1-1 with $\sigma_{j}^{\prime}=s_{j}$, then $f$ must be linear on a closed interval $\left[0, \max \left\{\sum s_{k} x_{k}, \sum_{i \neq j} s_{i} x_{i}+s_{j} x_{j}^{\prime}\right\}\right]$, and we obtain $g_{j}^{*}\left(\boldsymbol{s}, x_{j}^{\prime}, \boldsymbol{x}_{-j}, y_{j}^{\prime}, \boldsymbol{y}_{-j}\right) \leq y_{j}+s_{j} \cdot f^{\prime}\left(\sum s_{k} x_{k}\right) \cdot\left(x_{j}^{\prime}-x_{j}\right)$. In summary, by Assumption 1 and Pareto efficiency of $z$, no agent has an incentive to deviate from $(s, \boldsymbol{x}, \boldsymbol{y})$. 
(2) Show $N A\left(g^{*}, \boldsymbol{u}, \boldsymbol{s}\right) \subseteq P R(\boldsymbol{u}, \boldsymbol{s})$ for each $(\boldsymbol{u}, \boldsymbol{s}) \in \mathcal{E}$. Let $(\sigma, \boldsymbol{x}, \boldsymbol{y}) \in$ $N E\left(g^{*}, \boldsymbol{u}, \boldsymbol{s}\right)$.

Suppose that $(\boldsymbol{\sigma}, \boldsymbol{x}, \boldsymbol{y})$ corresponds to Rule 2. Let $N^{0}(\boldsymbol{x}) \equiv\left\{i \in N \mid x_{i}=0\right\}$. If $N^{0}(\boldsymbol{x})=\varnothing$, then $g_{i}^{*}(\boldsymbol{\sigma}, \boldsymbol{x}, \boldsymbol{y})=0$ for all $i \in N$. Then, there exists $j \in N$ such that $\sum_{i \neq j} \sigma_{i} x_{i} \neq \sum_{i \neq j} s_{i} x_{i}$. Thus, $g_{j}^{*}\left(\sigma_{j}^{\prime}, x_{j}^{\prime}, y_{j}^{\prime}, \sigma_{-j}, \boldsymbol{x}_{-j}, \boldsymbol{y}_{-j}\right)>0$ with $\sigma_{j}^{\prime}>\max \left\{\sigma_{i} \mid i \in N\right\}$ and $x_{j}^{\prime}=0$ under Rule 2. If $\# N^{0}(\boldsymbol{x}) \geq 2$, then for any $j \in N^{0}(\boldsymbol{x}), g_{j}^{*}\left(\sigma_{j}^{\prime}, x_{j}^{\prime}, y_{j}^{\prime}, \boldsymbol{\sigma}_{-j}, \boldsymbol{x}_{-j}, \boldsymbol{y}_{-j}\right)=f\left(\sum s_{k} x_{k}\right)$ by $x_{j}^{\prime}=0$ and $\sigma_{j}^{\prime}>$ $\max \left\{\sigma_{i} \mid i \neq j\right\}$ under Rule 2. If \# $N^{0}(\boldsymbol{x})=1$ and $\# N \backslash N^{0}(\boldsymbol{x}) \geq 2$, then there exists $j \in N \backslash N^{0}(\boldsymbol{x})$ such that $\sum_{i \in N \backslash\left(N^{0}(\boldsymbol{x}) \cup\{j\}\right)} \sigma_{i} x_{i} \neq \sum_{i \in N \backslash\left(N^{0}(\boldsymbol{x}) \cup\{j\}\right)} s_{i} x_{i}$. Thus, $g_{j}^{*}\left(\sigma_{j}^{\prime}, x_{j}^{\prime}, y_{j}^{\prime}, \sigma_{-j}, \boldsymbol{x}_{-j}, \boldsymbol{y}_{-j}\right)>0$ by $\sigma_{j}^{\prime}>\max \left\{\sigma_{i} \mid i \in N\right\}$ and $x_{j}^{\prime}=0$ under Rule 2. If $N^{0}(\boldsymbol{x})=\{i\}$ and $N \backslash N^{0}(\boldsymbol{x})=\{j\}$, then $g_{j}^{*}\left(\sigma_{j}^{\prime}, x_{j}^{\prime}, y_{j}^{\prime}, \boldsymbol{\sigma}_{-j}, \boldsymbol{x}_{-j}, \boldsymbol{y}_{-j}\right)>$ 0 by $\sigma_{j}^{\prime}=s_{j}, x_{j}^{\prime}=\frac{\bar{x}}{2}$, and $y_{j}^{\prime}>\max \left\{y_{i}, f\left(s_{j} x_{j}^{\prime}\right)\right\}$ under Rule 1-3. In summary, no profile of strategies can constitute a Nash equilibrium in Rule 2.

Suppose that $(\boldsymbol{\sigma}, \boldsymbol{x}, \boldsymbol{y})$ corresponds to Rule 1-3. Then, $g_{j}^{*}(\boldsymbol{\sigma}, \boldsymbol{x}, \boldsymbol{y})=0$ for some $j \in N$. If either $x_{j}=0$ or $\sigma_{j}=s_{j}$, then $g_{j}^{*}\left(\sigma_{j}^{\prime}, x_{j}^{\prime}, y_{j}^{\prime}, \boldsymbol{\sigma}_{-j}, \boldsymbol{x}_{-j}, \boldsymbol{y}_{-j}\right)>0$ by $\sigma_{j}^{\prime}=s_{j}, x_{j}^{\prime}=\pi\left(\boldsymbol{x}_{-j}\right)$, and $y_{j}^{\prime}>\max \left\{f\left(s_{j} x_{j}^{\prime}\right), \max \left\{y_{i} \mid i \neq j\right\}\right\}$ under Rule 1-3. If $x_{j}>0$ and $\sigma_{j} \neq s_{j}$, then $g_{j}^{*}\left(\sigma_{j}^{\prime}, x_{j}^{\prime}, y_{j}^{\prime}, \boldsymbol{\sigma}_{-j}, \boldsymbol{x}_{-j}, \boldsymbol{y}_{-j}\right)>0$ by $\sigma_{j}^{\prime}>\max \left\{\sigma_{i} \mid i \in N\right\}$ and $x_{j}^{\prime}=0$ under Rule 2 .

Suppose that $(\boldsymbol{\sigma}, \boldsymbol{x}, \boldsymbol{y})$ corresponds to Rule 1-2-1. Then, $g_{i}^{*}(\boldsymbol{\sigma}, \boldsymbol{x}, \boldsymbol{y})=0$ for each $i \in N$. Note $x_{j}=0$ for some $j \in N$ implies $N(\boldsymbol{\sigma}, \boldsymbol{x}, \boldsymbol{y})=\{j\}$ or $N(\boldsymbol{\sigma}, \boldsymbol{x}, \boldsymbol{y})=\varnothing$. Hence $\boldsymbol{x} \gg \boldsymbol{0}$. For each $j \in N$ with $\sigma_{j}=s_{j}, g_{j}^{*}\left(\sigma_{j}^{\prime}, x_{j}^{\prime}, y_{j}^{\prime}, \boldsymbol{\sigma}_{-j}, \boldsymbol{x}_{-j}, \boldsymbol{y}_{-j}\right)>0$ by $\sigma_{j}^{\prime}=s_{j}, x_{j}^{\prime}=\pi\left(\boldsymbol{x}_{-j}\right)$, and $y_{j}^{\prime}>\max \left\{f\left(s_{j} x_{j}^{\prime}\right), \max \left\{y_{i} \mid i \neq j\right\}\right\}$ under Rule 1-3 or Rule 1-2-2. Also, for each $j \in N$ with $\sigma_{j} \neq s_{j}, g_{j}^{*}\left(\sigma_{j}^{\prime}, x_{j}^{\prime}, y_{j}^{\prime}, \boldsymbol{\sigma}_{-j}, \boldsymbol{x}_{-j}, \boldsymbol{y}_{-j}\right)>0$ by $\sigma_{j}^{\prime}>\max \left\{\sigma_{i} \mid i \in N\right\}$ and $x_{j}^{\prime}=0$ under Rule 2 .

Suppose that $(\boldsymbol{\sigma}, \boldsymbol{x}, \boldsymbol{y})$ corresponds to Rule 1-2-2. Then, $N \backslash N(\boldsymbol{\sigma}, \boldsymbol{x}, \boldsymbol{y}) \neq \varnothing$, and $x_{i}>0$ and $g_{i}^{*}(\boldsymbol{\sigma}, \boldsymbol{x}, \boldsymbol{y})=0$ for each $i \in N \backslash N(\boldsymbol{\sigma}, \boldsymbol{x}, \boldsymbol{y})$. For each $j \in N \backslash N(\boldsymbol{\sigma}, \boldsymbol{x}, \boldsymbol{y})$ with $\sigma_{j}=s_{j}, g_{j}^{*}\left(\sigma_{j}^{\prime}, x_{j}^{\prime}, y_{j}^{\prime}, \sigma_{-j}, \boldsymbol{x}_{-j}, \boldsymbol{y}_{-j}\right)>0$ by $\sigma_{j}^{\prime}=s_{j}, x_{j}^{\prime}=\pi\left(\boldsymbol{x}_{-j}\right)$, and $y_{j}^{\prime}>\max \left\{f\left(s_{j} x_{j}^{\prime}\right), \max \left\{y_{i} \mid i \neq j\right\}\right\}$ under Rule 1-3. Also, for any $j \in$ $N \backslash N(\boldsymbol{\sigma}, \boldsymbol{x}, \boldsymbol{y})$ with $\sigma_{j} \neq s_{j}, g_{j}^{*}\left(\sigma_{j}^{\prime}, x_{j}^{\prime}, y_{j}^{\prime}, \boldsymbol{\sigma}_{-j}, \boldsymbol{x}_{-j}, \boldsymbol{y}_{-j}\right)>0$ by $\sigma_{j}^{\prime}>$ $\max \left\{\sigma_{i} \mid i \in N\right\}$ and $x_{j}^{\prime}=0$ under Rule 2 .

Thus, $(\boldsymbol{\sigma}, \boldsymbol{x}, \boldsymbol{y})$ corresponds to Rule 1-1. Then, $g^{*}(\boldsymbol{\sigma}, \boldsymbol{x}, \boldsymbol{y})=\boldsymbol{y}$, which implies $g_{i}^{*}(\boldsymbol{\sigma}, \boldsymbol{x}, \boldsymbol{y})=\frac{\sigma_{i} x_{i}}{\sum \sigma_{k} x_{k}} f\left(\sum s_{k} x_{k}\right)$ for each $i \in N$. By Assumption 1, $\boldsymbol{x} \gg \boldsymbol{0}$. Moreover, $f\left(\sum \sigma_{k} x_{k}\right)=f\left(\sum s_{k} x_{k}\right)$. Therefore, by Lemma $1, \sigma=s$. Since $(\boldsymbol{\sigma}, \boldsymbol{x}, \boldsymbol{y}) \in N E\left(g^{*}, \boldsymbol{u}, \boldsymbol{s}\right)$, for each $i \in N$ and each $\left(\sigma_{i}^{\prime}, x_{i}^{\prime}, y_{i}^{\prime}\right) \in \mathcal{S} \times[0, \bar{x}] \times \mathbb{R}_{+}$, $u_{i}\left(x_{i}, g_{i}^{*}(\boldsymbol{\sigma}, \boldsymbol{x}, \boldsymbol{y})\right) \geq u_{i}\left(x_{i}^{\prime}, g_{i}^{*}\left(\sigma_{i}^{\prime}, x_{i}^{\prime}, y_{i}^{\prime}, \boldsymbol{\sigma}_{-i}, \boldsymbol{x}_{-i}, \boldsymbol{y}_{-i}\right)\right)$. For each $i \in N, g_{i}^{*}$ $\left(\sigma_{i}^{\prime}, x_{i}^{\prime}, y_{i}^{\prime}, \sigma_{-i}, \boldsymbol{x}_{-i}, \boldsymbol{y}_{-i}\right) \leq y_{i}+s_{i} \cdot f^{\prime}\left(\sum s_{k} x_{k}\right) \cdot\left(x_{i}^{\prime}-x_{i}\right)$ by $\sigma_{i}^{\prime}=s_{i}$ and $y_{i}^{\prime}=0$ under Rule 1-2-2. Thus, $(\boldsymbol{x}, \boldsymbol{y})$ is Pareto efficient for $(\boldsymbol{u}, \boldsymbol{s})$, so that $(\boldsymbol{x}, \boldsymbol{y}) \in P R(\boldsymbol{u}, \boldsymbol{s})$. 
(3) Show $S N A\left(g^{*}, \boldsymbol{e}\right)=N A\left(g^{*}, \boldsymbol{e}\right)$ for each $\boldsymbol{e} \in \mathcal{E}$. By definition, $S N A\left(g^{*}, \boldsymbol{e}\right) \subseteq$ $N A\left(g^{*}, \boldsymbol{e}\right)$. Suppose $S N A\left(g^{*}, \boldsymbol{e}\right) \subsetneq N A\left(g^{*}, \boldsymbol{e}\right)$. Then, there exists $(\boldsymbol{\sigma}, \boldsymbol{x}, \boldsymbol{y})$ $\in N E\left(g^{*}, e\right)$ such that for some $T \subsetneq N$ and some $\left(\sigma_{i}^{\prime}, x_{i}^{\prime}, y_{i}^{\prime}\right)_{i \in T} \in \mathcal{S}^{\# T} \times[0, \bar{x}]^{\# T} \times$ $\mathbb{R}_{+}^{\# T}$, and each $j \in T$,

$$
u_{j}\left(x_{j}, g_{j}^{*}(\boldsymbol{\sigma}, \boldsymbol{x}, \boldsymbol{y})\right)<u_{j}\left(x_{j}^{\prime}, g_{j}^{*}\left(\left(\sigma_{i}^{\prime}, x_{i}^{\prime}, y_{i}^{\prime}\right)_{i \in T},\left(\sigma_{l}, x_{l}, y_{l}\right)_{l \in N \backslash T}\right)\right) .
$$

Since $(\boldsymbol{\sigma}, \boldsymbol{x}, \boldsymbol{y}) \in N E\left(g^{*}, \boldsymbol{e}\right)$ corresponds to Rule 1-1 as shown above, $(\boldsymbol{\sigma}, \boldsymbol{x}, \boldsymbol{y})$ is $P R$-consistent, so that $\boldsymbol{x} \gg \mathbf{0}$ under Assumption 1. Hence, $\boldsymbol{\sigma}=\boldsymbol{s}$ by Lemma 1. Note also that $T=N$ is eliminated by Pareto efficiency of $N A\left(g^{*}, \boldsymbol{e}\right)$. By construction of $g^{*}$, there is at most one agent who has a positive share of output under Rules 1-2-1, 1-2-2, 1-3, and 2. Since $g_{i}^{*}(\boldsymbol{s}, \boldsymbol{x}, \boldsymbol{y})>0$ for all $i \in N,\left(\left(\sigma_{i}^{\prime}, x_{i}^{\prime}, y_{i}^{\prime}\right)_{i \in T},\left(s_{l}, x_{l}, y_{l}\right)_{l \in N \backslash T}\right)$ corresponds to Rule 1-1 by Assumption 1. Then, $f$ must be linear on a closed interval $\left[0, \max \left\{\sum s_{k} x_{k}, \sum_{i \in T} \sigma_{i}^{\prime} x_{i}^{\prime}+\sum_{l \in N \backslash T} s_{l} x_{l}\right\}\right]$, and we obtain

$$
g_{j}^{*}\left(\left(\sigma_{i}^{\prime}, x_{i}^{\prime}, y_{i}^{\prime}\right)_{i \in T},\left(s_{l}, x_{l}, y_{l}\right)_{l \in N \backslash T}\right) \leq w_{j}+s_{j} \cdot f^{\prime}\left(\sum s_{k} x_{k}\right) \cdot\left(x_{j}^{\prime}-x_{j}\right)
$$

for some $j \in T$. Thus, by Pareto efficiency of $\left(\boldsymbol{x}, g^{*}(\boldsymbol{s}, \boldsymbol{x}, \boldsymbol{y})\right), N A\left(g^{*}, \boldsymbol{e}\right)=$ $\operatorname{SNA}\left(g^{*}, \boldsymbol{e}\right)$.

Proof of Theorem 2 Since $N A\left(g^{*}, \boldsymbol{e}\right)=P R(\boldsymbol{e})$ for each $\boldsymbol{e} \in \mathcal{E}$, we have only to show $P R(\boldsymbol{e}) \subseteq \operatorname{SPA}\left(\Gamma_{g^{*}}^{(\sigma, y) \circ x}, \boldsymbol{e}\right)$ for each $\boldsymbol{e} \in \mathcal{E}$. First, we show that in every Stage 2-subgame, there exists a Nash equilibrium. Let a strategy mapping $\chi^{*}: \mathcal{S}^{n} \times \mathbb{R}_{+}^{n} \rightarrow$ $[0, \bar{x}]^{n}$ be such that for each $\left(\Gamma_{g^{*}}^{(\sigma, y) \circ x}(\sigma, y), e\right)$ :

$$
\text { for all } i \in N, \chi_{i}^{*}(\boldsymbol{\sigma}, \boldsymbol{y})= \begin{cases}\overline{\bar{x}} & \text { if } y_{i}>\max \left\{y_{k} \mid k \neq i\right\}, \sigma_{i}=s_{i}, \text { and } \\ \hline \frac{\sigma}{2} & \text { otherwise }\end{cases}
$$

Note $g^{*}\left(\boldsymbol{\sigma}, \boldsymbol{\chi}^{*}(\boldsymbol{\sigma}, \boldsymbol{y}), \boldsymbol{y}\right)$ corresponds to Rule $\mathbf{1 - 3}$. To simplify the notation, let us denote $\boldsymbol{x}^{*}=\chi^{*}(\boldsymbol{\sigma}, \boldsymbol{y})$ in the following discussion.

Suppose that $i \in N$ switches from $x_{i}^{*}$ to $x_{i}^{\prime}$. Note that this deviation does not induce Rule 1-1. If $x_{i}^{\prime}$ induces Rule 1-2-2, then $g_{i}^{*}\left(\boldsymbol{\sigma}, x_{i}^{* \prime}, \boldsymbol{x}_{-i}^{*}, \boldsymbol{y}\right) \leq g_{i}^{*}\left(\boldsymbol{\sigma}, \boldsymbol{x}^{*}, \boldsymbol{y}\right)$, because $i \notin N\left(\sigma,\left(x_{i}^{\prime}, \boldsymbol{x}_{-i}^{*}\right), \boldsymbol{y}\right)$. If $x_{i}^{\prime}$ induces Rule 2 , then $x_{i}^{\prime}>0$, so that $g_{i}^{*}\left(\boldsymbol{\sigma}, x_{i}^{\prime}, \boldsymbol{x}_{-i}^{*}, \boldsymbol{y}\right)=$ 0. If $x_{i}^{\prime}$ induces Rule 1-3, then $g_{i}^{*}\left(\boldsymbol{\sigma}, x_{i}^{\prime}, \boldsymbol{x}_{-i}^{*}, \boldsymbol{y}\right) \leq g_{i}^{*}\left(\boldsymbol{\sigma}, \boldsymbol{x}^{*}, \boldsymbol{y}\right)$. To see this, let us assume that an agent, say $j$, has a positive output in $g^{*}\left(\sigma, x^{*}, \boldsymbol{y}\right)$. Then, $y_{j}>$ $\max \left\{y_{k} \mid k \neq j\right\}$. Since $\boldsymbol{x}^{*}=\left(\frac{\bar{x}}{2}, \mathbf{0}_{-j}\right)$, and $g^{*}\left(\boldsymbol{\sigma}, \boldsymbol{x}^{*}, \boldsymbol{y}\right)$ corresponds to Rule 1-3, $N\left(\boldsymbol{s},\left(\frac{\bar{x}}{2}, \mathbf{0}_{-j}\right), \boldsymbol{w}\right)=\varnothing$. Thus, if $i=j$, then $x_{i}^{*}=\frac{\bar{x}}{2}$ is the best response to $\mathbf{0}_{-i}$ in $\left(\Gamma_{g^{*}}^{(\boldsymbol{\sigma}, \boldsymbol{y}) \circ \boldsymbol{x}}(\boldsymbol{\sigma}, \boldsymbol{y}), \boldsymbol{e}\right)$. If $i \neq j$, then $g_{i}^{*}\left(\boldsymbol{\sigma}, x_{i}^{\prime}, \boldsymbol{x}_{-i}^{*}, \boldsymbol{y}\right)=0$. Thus, $\boldsymbol{x}^{*} \in$ $N E\left(\Gamma_{g^{*}}^{(\boldsymbol{\sigma}, \boldsymbol{y}) \circ \boldsymbol{x}}(\boldsymbol{\sigma}, \boldsymbol{y}), \boldsymbol{e}\right)$. 
Now, we will demonstrate that for each $\boldsymbol{e}=(\boldsymbol{u}, \boldsymbol{s}) \in \mathcal{E}$, if $\widehat{\boldsymbol{z}}=(\widehat{\boldsymbol{x}}, \widehat{\boldsymbol{y}}) \in P R(\boldsymbol{e})$, then there exists a subgame-perfect equilibrium whose corresponding outcome is $\widehat{z}$. Consider the following strategy profile of $\left(\Gamma_{g^{*}}^{(\sigma, y) \circ x}, \boldsymbol{e}\right)$ :

(1) In Stage $1,(\boldsymbol{\sigma}, \boldsymbol{y})=(\boldsymbol{s}, \widehat{\boldsymbol{y}})$.

(2) In Stage 2, $\chi: \mathcal{S}^{n} \times \mathbb{R}_{+}^{n} \rightarrow[0, \bar{x}]^{n}$ is given as follows:

$(2-1)$ : if $(\boldsymbol{\sigma}, \boldsymbol{y})=(\boldsymbol{s}, \widehat{\boldsymbol{y}})$ in Stage 1 , then $\chi(\sigma, \boldsymbol{y})=\widehat{\boldsymbol{x}}$;

$(2-2)$ : if $(\boldsymbol{\sigma}, \boldsymbol{y})=\left(\left(s_{j}^{\prime}, \boldsymbol{s}_{-j}\right),\left(y_{j}^{\prime}, \widehat{\boldsymbol{y}}_{-j}\right)\right)$ is such that $s_{j}^{\prime}=s_{j}, y_{j}^{\prime} \neq \widehat{y}_{j}$, and for each $i \neq j, y_{j}^{\prime} \geq y_{i}$ in Stage 1 , then for this $j \in N$,

$$
\chi_{j}(\boldsymbol{\sigma}, \boldsymbol{y})=\arg \max _{x_{j}^{\prime} \in[0, \bar{x}]} u_{j}\left(x_{j}^{\prime}, g_{j}^{*}\left(\boldsymbol{s}, x_{j}^{\prime}, \widehat{\boldsymbol{x}}_{-j}, y_{j}^{\prime}, \widehat{\boldsymbol{y}}_{-j}\right)\right)
$$

and for all $i \neq j, \chi_{i}(\boldsymbol{\sigma}, \boldsymbol{y})=\widehat{x}_{i}$;

(2-3): in any other case, $\chi(\sigma, y)=\chi^{*}(\sigma, y)$.

If the subgame $\left(\Gamma_{g^{*}}^{(\boldsymbol{\sigma}, \boldsymbol{y}) \circ \boldsymbol{x}}(\boldsymbol{\sigma}, \boldsymbol{y}), \boldsymbol{e}\right)$ corresponds to $(2-1)$, then $\chi(\boldsymbol{\sigma}, \boldsymbol{y}) \in N E$ $\left(\Gamma_{g^{*}}^{(\boldsymbol{\sigma}, \boldsymbol{y}) \circ \boldsymbol{x}}(\boldsymbol{\sigma}, \boldsymbol{y}), \boldsymbol{e}\right)$. This is because $(\widehat{\boldsymbol{x}}, \widehat{\boldsymbol{y}}) \in N A\left(g^{*}, \boldsymbol{e}\right)$ by Theorem 1. Also, by the above argument, $\chi(\sigma, y) \in N E\left(\Gamma_{g^{*}}^{(\sigma, y) \circ x}(\sigma, y), e\right)$ in the subgame (2-3) of Stage 2.

Suppose that $\left(\Gamma_{g^{*}}^{(\boldsymbol{\sigma}, \boldsymbol{y}) \circ \boldsymbol{x}}(\boldsymbol{\sigma}, \boldsymbol{y}), \boldsymbol{e}\right)$ corresponds to (2-2). Then, $g^{*}(\boldsymbol{\sigma}, \chi(\boldsymbol{\sigma}, \boldsymbol{y}), \boldsymbol{y})$ corresponds to Rule 1-1 or Rule 1-2, since $j \in N\left(s, x_{j}^{\prime}, \widehat{\boldsymbol{x}}_{-j}, y_{j}^{\prime}, \widehat{\boldsymbol{y}}_{-j}\right)$ or $P R\left(\boldsymbol{s}, x_{j}^{\prime}, \widehat{\boldsymbol{x}}_{-j}, y_{j}^{\prime}, \widehat{\boldsymbol{y}}_{-j}\right)^{-1} \neq \varnothing$ for any $x_{j}^{\prime} \in[0, \bar{x}]$. If $g^{*}(\boldsymbol{\sigma}, \chi(\boldsymbol{\sigma}, \boldsymbol{y}), \boldsymbol{y})$ corresponds to Rule 1-1, then $f$ must be linear on $\left[0, \max \left\{\sum s_{k} \widehat{x}_{k}, \sum_{i \neq j} s_{i} \widehat{x}_{i}+s_{j} x_{j}^{\prime}\right\}\right]$, which implies the Pareto efficiency of $\left(\left(x_{j}^{\prime}, \widehat{\boldsymbol{x}}_{-i}\right),\left(y_{j}^{\prime}, \widehat{\boldsymbol{y}}_{-j}\right)\right)$. Thus, $\chi(\boldsymbol{\sigma}, \boldsymbol{y}) \in$ $N E\left(\Gamma_{g^{*}}^{(\boldsymbol{\sigma}, \boldsymbol{y}) \circ \boldsymbol{x}}(\boldsymbol{\sigma}, \boldsymbol{y}), \boldsymbol{e}\right)$. If $g^{*}(\boldsymbol{\sigma}, \chi(\boldsymbol{\sigma}, \boldsymbol{y}), \boldsymbol{y})$ corresponds to Rule $\mathbf{1 - 2}$, then $\chi_{j}(\boldsymbol{\sigma}, \boldsymbol{y})=0$ is a best response to $\widehat{\boldsymbol{x}}_{-j}$ in $\left(\Gamma_{g^{*}}^{(\boldsymbol{\sigma}, \boldsymbol{y}) \circ \boldsymbol{x}}(\boldsymbol{\sigma}, \boldsymbol{y}), \boldsymbol{e}\right)$. This is because $N\left(\boldsymbol{s}, 0, \widehat{\boldsymbol{x}}_{-j}, y_{j}^{\prime}, \widehat{\boldsymbol{y}}_{-j}\right)=\{j\}$, and so $g^{*}(\boldsymbol{\sigma}, \boldsymbol{\chi}(\boldsymbol{\sigma}, \boldsymbol{y}), \boldsymbol{y})$ corresponds to Rule 1-2-2. For any other $i \neq j$, any deviation from $\widehat{x}_{i}$ to $x_{i}^{\prime}$ results in $g_{i}^{*}\left(\sigma, x_{i}^{\prime}, \chi_{-i}(\sigma, y), \boldsymbol{y}\right)=$ 0 . Thus, $\chi(\sigma, y) \in N E\left(\Gamma_{g^{*}}^{(\sigma, y) \circ x}(\sigma, y), e\right)$.

Now, let us see that the above strategy profile (1)-(2) constitutes a subgameperfect equilibrium of $\left(\Gamma_{g^{*}}^{(\boldsymbol{\sigma}, \boldsymbol{y}) \circ \boldsymbol{x}}, \boldsymbol{e}\right)$. By the strategy profile (1)-(2) of $\left(\Gamma_{g^{*}}^{(\boldsymbol{\sigma}, \boldsymbol{y}) \circ \boldsymbol{x}}, \boldsymbol{e}\right)$, $g^{*}(\boldsymbol{\sigma}, \chi(\boldsymbol{\sigma}, \boldsymbol{y}), \boldsymbol{y})=g^{*}(\boldsymbol{s}, \widehat{\boldsymbol{x}}, \widehat{\boldsymbol{y}})=\widehat{\boldsymbol{y}}$. Suppose that $j \in N$ switches from $\left(s_{j}, \widehat{y}_{j}\right)$ to $\left(s_{j}^{\prime}, y_{j}^{\prime}\right)$ in Stage 1 . Then by (2-2) and (2-3), $g_{j}^{*}\left(s_{j}^{\prime}, s_{-j}, \chi\left(s_{j}^{\prime}, s_{-j}, y_{j}^{\prime}, \widehat{\boldsymbol{y}}_{-j}\right), y_{j}^{\prime}\right.$, $\left.\widehat{\boldsymbol{y}}_{-j}\right) \leq \widehat{y}_{j}+s_{j} \cdot f^{\prime}\left(\sum s_{k} \widehat{x}_{k}\right) \cdot\left(x_{j}^{\prime}-\widehat{x}_{j}\right)$. Thus, since $(\widehat{\boldsymbol{x}}, \widehat{\boldsymbol{y}}) \in P R(\boldsymbol{e}),(\widehat{\boldsymbol{x}}, \widehat{\boldsymbol{y}}) \in$ $S P A\left(\Gamma_{g^{*}}^{(\boldsymbol{\sigma}, \boldsymbol{y}) \circ \boldsymbol{x}}, \boldsymbol{e}\right)$. 
Proof of Theorem 3 Since $N A\left(g^{*}, \boldsymbol{e}\right)=P R(\boldsymbol{e})$ for each $\boldsymbol{e} \in \mathcal{E}$, we have only to show $P R(\boldsymbol{e}) \subseteq S P A\left(\Gamma_{g^{*}}^{x \circ(\sigma, y)}, \boldsymbol{e}\right)$. First, we will show that in every Stage 2-subgame, there is a Nash equilibrium.

Given $\boldsymbol{e}=(\boldsymbol{u}, \boldsymbol{s}) \in \mathcal{E}$, let a strategy mapping $\boldsymbol{\mu}^{*}:[0, \bar{x}]^{n} \rightarrow \mathcal{S}^{n} \times \mathbb{R}_{+}^{n}$ be such that for each $\left(\Gamma_{g^{*}}^{x \circ(\sigma, y)}(\boldsymbol{x}), \boldsymbol{e}\right), \boldsymbol{\mu}^{*}(\boldsymbol{x})=\left(\sigma^{*}, \boldsymbol{y}^{*}\right)$, where for each $i \in N$ :

$$
\left(\sigma_{i}^{*}, y_{i}^{*}\right)= \begin{cases}\left(s_{i}, 0\right) & \text { if } x_{i} \neq \pi\left(\boldsymbol{x}_{-i}\right) \\ \left(s_{i}, f\left(s_{i} x_{i}\right)+1\right) & \text { otherwise }\end{cases}
$$

Suppose that $i \in N$ switches from $\left(\sigma_{i}^{*}, y_{i}^{*}\right)$ to $\left(\sigma_{i}^{\prime}, y_{i}^{\prime}\right)$. Note that $g^{*}\left(\boldsymbol{\sigma}^{*}, \boldsymbol{x}, \boldsymbol{y}^{*}\right)$ corresponds to Rule 1-3. Thus, $i$ cannot induce Rule 1-1 by changing his strategy. If $\left(\sigma_{i}^{\prime}, y_{i}^{\prime}\right)$ induces Rule 1-2-2, then $g_{i}^{*}\left(\sigma_{i}^{\prime}, \boldsymbol{\sigma}_{-i}^{*}, \boldsymbol{x}, y_{i}^{\prime}, \boldsymbol{y}_{-i}^{*}\right)=0$, because $i \notin N\left(\sigma_{i}^{\prime}, \boldsymbol{\sigma}_{-i}^{*}, \boldsymbol{x}, y_{i}^{\prime}, \boldsymbol{y}_{-i}^{*}\right)$. If $\left(\sigma_{i}^{\prime}, y_{i}^{\prime}\right)$ induces Rule 2 , then $x_{i}>0$, which implies $g_{i}^{*}\left(\sigma_{i}^{\prime}, \boldsymbol{\sigma}_{-i}^{*}, \boldsymbol{x}, y_{i}^{\prime}, \boldsymbol{y}_{-i}^{*}\right)=0$. If $\left(\sigma_{i}^{\prime}, y_{i}^{\prime}\right)$ induces Rule 1-3, then $g_{i}^{*}\left(\sigma_{i}^{\prime}, \boldsymbol{\sigma}_{-i}^{*}, \boldsymbol{x}\right.$, $\left.y_{i}^{\prime}, \boldsymbol{y}_{-i}^{*}\right) \leq g_{i}^{*}\left(\boldsymbol{\sigma}^{*}, \boldsymbol{x}, \boldsymbol{y}^{*}\right)$, since whether $x_{i}=\pi\left(\boldsymbol{x}_{-i}\right)$ or not is already fixed in Stage 1. Thus, $\left(\boldsymbol{\sigma}^{*}, \boldsymbol{y}^{*}\right) \in N E\left(\Gamma_{g^{*}}^{x \circ(\boldsymbol{\sigma}, \boldsymbol{y})}(\boldsymbol{x}), \boldsymbol{e}\right)$.

Now, we will show that if $\widehat{\boldsymbol{z}}=(\widehat{\boldsymbol{x}}, \widehat{\boldsymbol{y}}) \in P R(\boldsymbol{e})$, then there exists a subgame-perfect equilibrium whose corresponding outcome is $\widehat{z}$. Consider the following strategy profile of $\left(\Gamma_{g^{*}}^{x \circ(\sigma, y)}, e\right)$ :

(1) In Stage 1 , each $i \in N$ supplies $\widehat{x}_{i}>0$.

(2) In Stage 2, $\boldsymbol{\mu}:[0, \bar{x}]^{n} \rightarrow \mathcal{S}^{n} \times \mathbb{R}_{+}^{n}$ is given as follows:

$(2-1)$ : if $\boldsymbol{x}=\widehat{\boldsymbol{x}}$ in Stage 1, then $\boldsymbol{\mu}(\boldsymbol{x})=(\boldsymbol{s}, \widehat{\boldsymbol{y}})$;

$(2-2)$ : if $\boldsymbol{x}=\left(x_{j}^{\prime}, \widehat{\boldsymbol{x}}_{-j}\right) \gg \mathbf{0}$, where $x_{j}^{\prime} \neq \widehat{x}_{j}$ in Stage 1 , then

$$
\begin{aligned}
\text { for } j \in N, \mu_{j}(\boldsymbol{x}) & =\left(s_{j}, f\left(s_{j} x_{j}^{\prime}\right)+1\right), \\
\text { for all } i \neq j, \mu_{i}(\boldsymbol{x}) & = \begin{cases}\left(s_{i}, \widehat{y}_{i}\right) & \text { if } x_{i} \neq \pi\left(\boldsymbol{x}_{-i}\right) \\
\left(s_{i}, f\left(\sum_{k \neq j} s_{k} \widehat{x}_{k}+s_{j} x_{j}^{\prime}\right)\right) & \text { otherwise }\end{cases}
\end{aligned}
$$

(2-3): in any other case, $\boldsymbol{\mu}(\boldsymbol{x})=\boldsymbol{\mu}^{*}(\boldsymbol{x})$.

If the subgame $\left(\Gamma_{g^{*}}^{x \circ(\sigma, y)}(\boldsymbol{x}), \boldsymbol{e}\right)$ corresponds to $(2-1)$, then $(\boldsymbol{s}, \widehat{\boldsymbol{y}}) \in N E$ $\left(\Gamma_{g^{*}}^{x \circ(\sigma, y)}(\boldsymbol{x}), \boldsymbol{e}\right)$. This is because $(\widehat{\boldsymbol{x}}, \widehat{\boldsymbol{y}}) \in N A\left(g^{*}, \boldsymbol{e}\right)$ by Theorem 1. Also, by the above argument, $\boldsymbol{\mu}^{*}(\boldsymbol{x}) \in N E\left(\Gamma_{g^{*}}^{x \circ(\sigma, y)}(\boldsymbol{x}), \boldsymbol{e}\right)$ in the subgame (2-3) of Stage 2.

Suppose that $\left(\Gamma_{g^{*}}^{x \circ(\sigma, y)}(\boldsymbol{x}), \boldsymbol{e}\right)$ corresponds to (2-2), and $h \in N$ switches from $\mu_{h}(\boldsymbol{x})$ to $\left(s_{h}^{\prime}, y_{h}^{\prime}\right)$. First, if $s_{h}^{\prime} \neq s_{h}$, then $g_{h}^{*}\left(s_{h}^{\prime}, y_{h}^{\prime}, \boldsymbol{\mu}_{-h}(\boldsymbol{x}), \boldsymbol{x}\right)=0$ under Rule 2. Secondly, consider the following two cases:

(i) For all $i \neq j, x_{i} \neq \pi\left(\boldsymbol{x}_{-i}\right)$. Then, $g^{*}(\boldsymbol{\mu}(\boldsymbol{x}), \boldsymbol{x})$ corresponds to Rule 1-2-2. If $h \neq j$ and $\left(s_{h}^{\prime}, y_{h}^{\prime}\right)$ induces Rule 1-2-2 or Rule 1-3, then $g_{h}^{*}\left(s_{h}^{\prime}, y_{h}^{\prime}, \boldsymbol{\mu}_{-h}(\boldsymbol{x}), \boldsymbol{x}\right)=0$. Note if $h \neq j$, then $\left(s_{h}^{\prime}, y_{h}^{\prime}\right)$ cannot induce Rule 1-1. If $h=j$ and $\left(s_{h}^{\prime}, y_{h}^{\prime}\right)$ induces 
Rule 1-2-2 or Rule 1-1, then $g_{h}^{*}\left(s_{h}^{\prime}, y_{h}^{\prime}, \boldsymbol{\mu}_{-h}(\boldsymbol{x}), \boldsymbol{x}\right) \leq \widehat{y}_{h}+s_{h} \cdot f^{\prime}\left(\sum s_{k} \widehat{x}_{k}\right)$. $\left(x_{h}^{\prime}-\widehat{x}_{h}\right)$. Note if $h=j$, then $\left(s_{h}^{\prime}, y_{h}^{\prime}\right)$ cannot induce Rule 1-3. Thus, $\boldsymbol{\mu}(\boldsymbol{x}) \in$ $N E\left(\Gamma_{g^{*}}^{\boldsymbol{x} \circ(\boldsymbol{\sigma}, \boldsymbol{y})}(\boldsymbol{x}), \boldsymbol{e}\right)$.

(ii) There exists $i^{*} \neq j$ with $x_{i^{*}}=\pi\left(\boldsymbol{x}_{-i^{*}}\right)$. Then, $g^{*}(\boldsymbol{\mu}(\boldsymbol{x}), \boldsymbol{x})$ corresponds to Rule 1-3. Then, $\left(s_{h}^{\prime}, y_{h}^{\prime}\right)$ cannot induce Rule 1-1. If $\left(s_{h}^{\prime}, y_{h}^{\prime}\right)$ induces Rule 1-2-2, this implies $h=i^{*}$ or $h=j$. Thus, since $h \notin N\left(s_{h}^{\prime}, y_{h}^{\prime}, \boldsymbol{\mu}_{-h}(\boldsymbol{x}), \boldsymbol{x}\right)$, $g_{h}^{*}\left(s_{h}^{\prime}, y_{h}^{\prime}, \boldsymbol{\mu}_{-h}(\boldsymbol{x}), \boldsymbol{x}\right)=0$. If $\left(s_{h}^{\prime}, y_{h}^{\prime}\right)$ induces Rule 1-3, then $g_{h}^{*}\left(s_{h}^{\prime}, y_{h}^{\prime}, \boldsymbol{\mu}_{-h}(\boldsymbol{x}), \boldsymbol{x}\right)$ $\leq g^{*}(\boldsymbol{\mu}(\boldsymbol{x}), \boldsymbol{x})$, since $x_{i^{*}}=\pi\left(\boldsymbol{x}_{-i^{*}}\right)$ is already fixed in Stage 1. Thus, $\boldsymbol{\mu}(\boldsymbol{x}) \in$ $N E\left(\Gamma_{g^{*}}^{x \circ(\sigma, y)}(\boldsymbol{x}), \boldsymbol{e}\right)$.

Now, let us see that the above strategy profile (1)-(2) constitutes a subgameperfect equilibrium of $\left(\Gamma_{g^{*}}^{x \circ(\sigma, y)}, \boldsymbol{e}\right)$. By the strategy profile (1)-(2) of $\left(\Gamma_{g^{*}}^{x \circ(\sigma, y)}, \boldsymbol{e}\right)$, $g^{*}(\boldsymbol{\mu}(\widehat{\boldsymbol{x}}), \widehat{\boldsymbol{x}})=g^{*}(\boldsymbol{s}, \widehat{\boldsymbol{x}}, \widehat{\boldsymbol{y}})=\widehat{\boldsymbol{y}}$. Suppose that $j \in N$ deviates from $\widehat{x}_{j}$ to $x_{j}^{\prime} \neq \widehat{x}_{j}$ in Stage 1. If $x_{j}^{\prime}=0$, then by (2-3), $g_{j}^{*}\left(\boldsymbol{\mu}\left(x_{j}^{\prime}, \widehat{\boldsymbol{x}}_{-j}\right),\left(x_{j}^{\prime}, \widehat{\boldsymbol{x}}_{-j}\right)\right)=0$. If $x_{j}^{\prime}>0$, then by $(2-2), g_{j}^{*}\left(\boldsymbol{\mu}\left(x_{j}^{\prime}, \widehat{\boldsymbol{x}}_{-j}\right),\left(x_{j}^{\prime}, \widehat{\boldsymbol{x}}_{-j}\right)\right) \leq \widehat{y}_{j}+s_{j} \cdot f^{\prime}\left(\sum s_{k} \widehat{x}_{k}\right) \cdot\left(x_{j}^{\prime}-\widehat{x}_{j}\right)$. Thus, since $(\widehat{\boldsymbol{x}}, \widehat{\boldsymbol{y}}) \in P R(\boldsymbol{e}),(\widehat{\boldsymbol{x}}, \widehat{\boldsymbol{y}}) \in \operatorname{SPA}\left(\Gamma_{g^{*}}^{x \circ(\sigma, y)}, \boldsymbol{e}\right)$.

Open Access This article is distributed under the terms of the Creative Commons Attribution Noncommercial License which permits any noncommercial use, distribution, and reproduction in any medium, provided the original author(s) and source are credited.

\section{References}

Corchón LC, Puy MS (2002) Existence and Nash implementation of efficient sharing rules for a commonly owned technology. Soc Choice Welf 19:369-379

Hong L (1995) Nash implementation in production economies. Econ Theory 5:401-417

Hurwicz L (1960) Optimality and informational efficiency in resource allocation processes. In: Arrow KJ, Karlin S, Suppes P (eds) Mathematical methods in social sciences. Stanford University Press, Stanford pp 27-46

Hurwicz L, Maskin E, Postlewaite A (1995) Feasible Nash implementation of social choice rules when the designer does not know endowments or production sets. In: Ledyard J (ed) The economics of informational decentralization: complexity, efficiency, and stability. Essays in Honor of Stanley Reiter. Kluwer, Amsterdam pp 367-433

Jackson MO, Palfrey T, Srivastava S (1994) Undominated Nash implementation in bounded mechanisms. Games Econ Behav 6:474-501

Kaplan T, Wettstein D (2000) Surplus sharing with a two-stage mechanism. Int Econ Rev 41:399-409

Kranich L (1994) Equal division, efficiency, and the sovereign supply of labor. Am Econ Rev 84:178-189

Roemer J (1989) A public ownership resolution of the tragedy of the commons. Soc Philos Policy 6:74-92

Roemer J, Silvestre J (1993) The proportional solution for economies with both private and public ownership. J Econ Theory 59:426-444

Saijo T, Tatamitani Y, Yamato T (1996) Toward natural implementation. Int Econ Rev 37:949-980

Suh S-C (1995) A mechanism implementing the proportional solution. Econ Des 1:301-317

Tian G (1999) Double implementation in economies with production technologies unknown to the designer. Econ Theory 13:689-707

Tian G (2000) Incentive mechanism design for production economies with both private and public ownerships. Games Econ Behav 33:294-320

Varian H (1994) A solution to the problem of externalities when agents are well informed. Am Econ Rev $84: 1278-1293$ 
Yoshihara N (1999) Natural and double implementation of public ownership solutions in differentiable production economies. Rev Econ Des 4:127-151

Yoshihara N (2000a) A characterization of natural and double implementation in production economies. Soc Choice Welf 17:571-599

Yoshihara N (2000b) On efficient and procedurally-fair equilibrium allocations in sharing games. Institute of Economic Research Discussion Paper Series A No. 397. Hitotsubashi University 